\title{
CANDELS: THE CONTRIBUTION OF THE OBSERVED GALAXY POPULATION TO COSMIC REIONIZATION
}

\author{
Steven L. Finkelstein ${ }^{1,11}$, Casey Papovich ${ }^{2}$, Russell E. Ryan Jr. ${ }^{3}$, Andreas H. Pawlik ${ }^{1}$, Mark Dickinson ${ }^{4}$, \\ Henry C. Ferguson ${ }^{3}$, Kristian Finlator ${ }^{5,11}$, Anton M. Koekemoer ${ }^{3}$, Mauro Giavalisco ${ }^{6}$, Asantha Cooray ${ }^{7}$, \\ James S. Dunlor ${ }^{8}$, SAndy M. Faber ${ }^{9}$, Norman A. Grogin ${ }^{3}$, Dale D. KoceVsKi ${ }^{9}$, and JeFFrey A. Newman ${ }^{10}$ \\ ${ }^{1}$ Department of Astronomy, The University of Texas at Austin, Austin, TX 78712, USA; stevenf@astro.as.utexas.edu \\ ${ }^{2}$ George P. and Cynthia Woods Mitchell Institute for Fundamental Physics and Astronomy, \\ Department of Physics and Astronomy, Texas A\&M University, College Station, TX 77843, USA \\ ${ }^{3}$ Space Telescope Science Institute, Baltimore, MD 21218, USA \\ ${ }^{4}$ National Optical Astronomy Observatory, Tucson, AZ 85719, USA \\ ${ }^{5}$ Physics Department, University of California, Santa Barbara, CA 93106, USA \\ ${ }^{6}$ Department of Astronomy, University of Massachusetts, Amherst, MA 01003, USA \\ ${ }^{7}$ Department of Physics and Astronomy, University of California, Irvine, CA 92697, USA \\ ${ }^{8}$ Institute for Astronomy, University of Edinburgh, Royal Observatory, Edinburgh, UK \\ ${ }^{9}$ University of California Observatories/Lick Observatory, University of California, Santa Cruz, CA 95064, USA \\ ${ }^{10}$ Department of Physics and Astronomy and Pitt-PACC, University of Pittsburgh, Pittsburgh, PA 15260, USA \\ Received 2012 June 4; accepted 2012 August 12; published 2012 October 4
}

\begin{abstract}
We present measurements of the specific ultraviolet luminosity density from a sample of 483 galaxies at $6 \lesssim z \lesssim 8$. These galaxies were selected from new deep near-infrared Hubble Space Telescope imaging from the Cosmic Assembly Near-infrared Deep Extragalactic Legacy Survey, Hubble UltraDeep Field 2009, and Wide Field Camera 3 Early Release Science programs. We investigate the contribution to reionization from galaxies that we observe directly, thus sidestepping the uncertainties inherent in complementary studies that have invoked assumptions regarding the intrinsic shape or the faint-end cutoff of the galaxy ultraviolet (UV) luminosity function. Due to our larger survey volume, wider wavelength coverage, and updated assumptions about the clumping of gas in the intergalactic medium (IGM), we find that the observable population of galaxies can sustain a fully reionized IGM at $z=6$, if the average ionizing photon escape fraction $\left(f_{\mathrm{esc}}\right)$ is $\sim 30 \%$. Our result contrasts with a number of previous studies that have measured UV luminosity densities at these redshifts that vary by a factor of five, with many concluding that galaxies could not complete reionization by $z=6$ unless a large population of galaxies fainter than the detection limit were invoked, or extremely high values of $f_{\text {esc }}$ were present. The specific UV luminosity density from our observed galaxy samples at $z=7$ and 8 is not sufficient to maintain a fully reionized IGM unless $f_{\text {esc }}>50 \%$. We examine the contribution from galaxies in different luminosity ranges and find that the sub- $L^{*}$ galaxies we detect are stronger contributors to the ionizing photon budget than the $L>L^{*}$ population, unless $f_{\text {esc }}$ is luminosity dependent. Combining our observations with constraints on the emission rate of ionizing photons from Ly $\alpha$ forest observations at $z=6$, we find that we can constrain $f_{\text {esc }}<34 \%(2 \sigma)$ if the observed galaxies are the only contributors to reionization, or $<13 \%(2 \sigma)$ if the luminosity function extends to a limiting magnitude of $M_{\mathrm{UV}}=-13$. These escape fractions are sufficient to sustain an ionized IGM by $z=6$. Current constraints on the high-redshift galaxy population imply that the volume ionized fraction of the IGM, while consistent with unity at $z \leqslant 6$, appears to drop at redshifts not much higher than 7 , consistent with a number of complementary reionization probes. If faint galaxies dominated the ionizing photon budget at $z=6-7$, future extremely deep observations with the James Webb Space Telescope will probe deep enough to directly observe them, providing an indirect constraint on the global ionizing photon escape fraction.
\end{abstract}

Key words: early universe - galaxies: evolution - galaxies: formation - galaxies: high-redshift ultraviolet: galaxies

Online-only material: color figures

\section{INTRODUCTION}

The reionization of the intergalactic medium (IGM) is the last major phase transition in the universe, and as such has been a major topic of recent study. Over the past decade, a number of lines of evidence have yielded improved constraints on the duration of reionization, the completion redshift, and the primary source of ionizing photons.

Observations of the cosmic microwave background measure the Thomson scattering optical depth due to electrons in the path. The updated 7 year Wilkinson Microwave Anisotropy Probe

\footnotetext{
${ }^{11}$ Hubble Fellow.
}

(WMAP) results measure $\tau=0.088 \pm 0.014$, which corresponds to a redshift of instantaneous reionization of $z=10.6 \pm 1.2$ (Komatsu et al. 2011). However, reionization is likely to be a much more extended event, especially if faint galaxies play a dominant role. Measurements of the kinetic Sunyaev-Zeldovich (kSZ) effect with the South Pole Telescope (SPT) from Zahn et al. (2012) have recently placed a limit on the duration of reionization, of $\Delta z<7.9$. When combined with the WMAP constrains on instantaneous reionization, they conclude at $95 \%$ confidence that reionization was complete at $z>5.8$, though this measurement may not be sensitive to an extended end to reionization. Additionally, observations of the Ly $\alpha$ forest in high-redshift quasars suggest an end to reionization at $z \sim 6$, 
as the measurements of the near-zones around $z=6$ quasars yield results consistent with a negligible neutral fraction (e.g., Fan et al. 2006). However, this relies on the assumption of a uniform background, which is likely not the case. A recent model-independent analysis claims an upper limit on the ionized fraction of the IGM at $z=6$ of only $\sim 50 \%$ (McGreer et al. 2011). More $z>6$ quasar sight lines may be needed to conclusively verify both results (Mesinger 2010; see also Becker et al. 2007).

Nonetheless, some sources must be responsible for reionizing the IGM at $z \gtrsim 6$. Quasars were a natural choice, as they are extremely bright, and the bulk of their ionizing photons can escape. However, the quasar luminosity function peaks at $z \sim 2$ and falls off rapidly toward higher redshift (e.g., Hopkins et al. 2007), with recent measurements at $z \sim 6$ showing that the quasar ionizing flux is insufficient by more than a factor of 10 of what is needed to reionize the IGM (Willott et al. 2010). Additionally, observations of the X-ray background rule out a dominant contribution to the reionizing photon budget from quasars (Dijkstra et al. 2004a). Finally, if quasars dominated the reionization of hydrogen, one would expect helium to be reionized in the same epoch, which is not the case (e.g., McQuinn 2012).

Population III stars, due to their predicted high masses (e.g., Bromm \& Larson 2004; Glover 2005), were very efficient emitters of ionizing photons (Tumlinson \& Shull 2000; Bromm et al. 2001; Schaerer 2002). Their overall contribution to reionization may, however, have been limited (e.g., Greif \& Bromm 2006). This conclusion has been strengthened by recent simulation results which have corrected the mass scale of the first stars down to less extreme values (Clark et al. 2011; Greif et al. 2011, 2012).

The most likely source for the bulk of ionizing photons is thus from star-forming galaxies themselves, where ionizing photons are created from the massive stars present during ongoing star formation. This has been well-studied observationally, but it was only in the last decade when large samples of galaxies close to the reionization epoch were compiled. Following wide and deep surveys with the Advanced Camera for Surveys (ACS) on board the Hubble Space Telescope (HST), large samples of $z \sim 6$ galaxies were compiled (e.g., Giavalisco et al. 2004; Bunker et al. 2004). While Giavalisco et al. (2004) found that the specific UV luminosity density (and thus the star formation rate density) was roughly constant from $z=4$ to 6 , Bunker et al. (2004) found, via deeper observations, that this quantity appeared to decline toward higher redshift. Using the commonly accepted (at the time) large value for the clumping factor of the IGM, they concluded that galaxies were unable to account for the necessary ionizing photons to reionize the universe. Yan $\&$ Windhorst (2004) pointed out that if the faint-end slope of the galaxy luminosity function was steep, it could be that $L<0.1 L^{*}$ galaxies dominated the ionizing photon budget, and thus allowed galaxies to complete reionization by $z=6$. Regardless of the interpretation, these galaxy samples contained only $\sim 50-100$ galaxies, each of which was only detected in one band, given the lack of deep HST near-infrared observations at the time.

The advent of the Wide Field Camera 3 (WFC3) on HST has opened the door to the $z \geqslant 6$ universe, allowing the first investigation into whether reionization completed at $z=7$ or earlier. This is combined with recent results which have revised the expected IGM clumping factor to much lower values (see Section 4.1). Finkelstein et al. (2010) investigated the combined UV luminosities of their observed $z=7$ galaxies and found that while they could come close to reionizing the IGM, either fainter galaxies or high $(\geqslant 50 \%)$ escape fractions were necessary to complete reionization by $z=7$ (see also Bunker et al. 2010)

A number of studies have used measured luminosity functions of $z \sim 7$ and 8 galaxies to infer their contribution to reionization, by integrating them down to an assumed magnitude limit. The majority of these studies found that, once galaxies fainter than the detection threshold are accounted for, galaxies could reionize the universe at such early times (e.g., Oesch et al. 2010b; McLure et al. 2010; Lorenzoni et al. 2011; Bouwens et al. 2012; see also Grazian et al. 2011). This is however reliant on a number of assumptions. First, the luminosity function results are susceptible to the assumption of a Schechter function parameterization. At $z \sim 5-6$, where the samples are larger, there is no strong evidence for deviation from this function (e.g., Bouwens et al. 2007; McLure et al. 2009). However, as we push closer to the Big Bang, galaxies are changing rapidly, thus at some point we may encounter an epoch where the Schechter function is no longer an accurate representation. This could be due to a variety of effects, with one being a lack of active galactic nuclei (AGNs) feedback if AGNs are not yet present in the centers of all galaxies at very high redshifts, which is plausible depending on the speed with which supermassive black holes form in the early universe.

Additionally, while recent evidence has indicated a very steep faint end slope at $z \geqslant 7$ (e.g., Bouwens et al. 2011a; Oesch et al. 2012; Bradley et al. 2012), the uncertainty on these measurements are large (e.g., $\sigma(\alpha) \approx 0.2$ at $z=8$; Bradley et al. 2012). Finally, when integrating the luminosity function, one needs to choose a limiting magnitude, as suppression from the UV background will result in the gas in galaxies becoming heated below some circular velocity (i.e., mass) limit. Galaxies above this limit are dense enough where collisional excitation of $\mathrm{H}$ I by electrons can overcome photoionization heating, and have their gas cool and form stars. However, we have no observational evidence for this value, and theoretical results from the literature yield values of $-15<M_{\mathrm{lim}}<-10$ (e.g., Finlator et al. 2011; Muñoz \& Loeb 2011; Kulkarni \& Choudhury 2011; Choudhury et al. 2008). With a steep faint-end slope, a difference of this level can result in a difference in the integrated luminosity density by more than a factor of two.

Here, we measure the specific luminosity density of the observable galaxy population using the largest sample of $6<$ $z<8$ galaxies yet compiled, which allows us to study the contribution of galaxies to reionization without the uncertainties inherent in invoking a parameterized luminosity function. This allows us to assess whether a significant contribution from galaxies below the observational limits is necessary to sustain an ionized IGM at a given redshift. We emphasize our results at $z=6$, where we have a large sample, and each galaxy is detected in four individual imaging bands, yielding much more robust results over the previous studies which had access only to optical ACS data.

In Section 2, we describe the data sets used in this study, as well as our photometry and sample selection methods. We discuss how we measured the rest-frame UV specific luminosity density, and corrected it for incompleteness down to our magnitude limit in Section 3, while in Section 4, we discuss the implications of our results on the reionization of the IGM. Throughout this paper we assume a concordance cosmology, with $H_{\mathrm{o}}=70 \mathrm{~km} \mathrm{~s}^{-1} \mathrm{Mpc}^{-1}, \Omega_{m}=0.3$, and $\Omega_{\lambda}=0.7$. All quoted volumes are co-moving, and all magnitudes are reported in the $\mathrm{AB}$ system, where $m_{\mathrm{AB}}=31.4-2.5 \log \left(f_{v} / 1 \mathrm{nJy}\right)$ (Oke $\&$ Gunn 1983). In the remainder of the paper, we make use of 
Table 1

Observations Summary

\begin{tabular}{|c|c|c|c|c|c|c|c|c|c|c|}
\hline Field & $\begin{array}{c}\text { Area } \\
\left(\operatorname{arcmin}^{2}\right)\end{array}$ & $\begin{array}{c}B_{435} \\
(\mathrm{mag})\end{array}$ & $\begin{array}{c}V_{606} \\
(\mathrm{mag})\end{array}$ & $\begin{array}{c}i_{775} \\
(\mathrm{mag})\end{array}$ & $\begin{array}{c}I_{814} \\
(\mathrm{mag})\end{array}$ & $\begin{array}{c}z 850 \\
\text { (mag) }\end{array}$ & $\begin{array}{c}Y_{098} \\
(\mathrm{mag})\end{array}$ & $\begin{array}{c}Y_{105} \\
(\mathrm{mag})\end{array}$ & $\begin{array}{c}J_{125} \\
(\mathrm{mag})\end{array}$ & $\begin{array}{l}H_{160} \\
(\mathrm{mag})\end{array}$ \\
\hline HUDF09 MAIN & 5.0 & 29.5 & 29.9 & 29.7 & $\cdots$ & 29.0 & $\cdots$ & 29.2 & 29.5 & 29.5 \\
\hline HUDF09 PAR1 & 4.4 & $\ldots$ & 28.9 & 28.7 & $\ldots$ & 28.6 & $\ldots$ & 28.0 & 28.9 & 28.7 \\
\hline HUDF09 PAR2 & 4.6 & $\cdots$ & 29.0 & 28.7 & $\cdots$ & 28.4 & $\cdots$ & 28.7 & 29.1 & 28.9 \\
\hline GOODS-S DEEP & 62.0 & 28.1 & 28.3 & 27.7 & 28.2 & 27.5 & $\ldots$ & 28.2 & 28.1 & 27.9 \\
\hline GOODS-S WIDE & 34.1 & 28.1 & 28.3 & 27.7 & 27.8 & 27.5 & $\cdots$ & 27.4 & 27.6 & 27.3 \\
\hline GOODS-S ERS & 40.5 & 28.1 & 28.3 & 27.7 & 27.7 & 27.5 & 27.6 & $\cdots$ & 28.0 & 27.7 \\
\hline
\end{tabular}

Notes. The area is measured from the image area used to detect objects, which excludes noisy regions on the edges, regions without data from all available filters, as well as regions overlapping deeper data (i.e., the CANDELS Deep field also covers the HUDF, thus we have masked out this region in the CANDELS imaging for this analysis). The remaining columns are $5 \sigma$ limiting magnitudes measured in a 0.4 diameter apertures on non-PSF matched images. The F814W data from CANDELS were only used in the visual inspection of $z=8$ candidate galaxies, primarily in the GOODS-S DEEP field as it is $\sim 0.5$ mag deeper than the existing GOODS ACS data.

luminosity functions from the literature over the redshift range of our study. We use the luminosity functions from Bouwens et al. (2007) for $z=4,5$, and 6; Bouwens et al. (2011a) for $z=7$; Bradley et al. (2012) for $z=8$, and Bouwens et al. (2011b) for $z=10$. We note that the analysis by Bradley et al. (2012) includes WFC3 pure parallel imaging from the Brightest of Reionizing Galaxies (Trenti et al. 2011) survey, resulting in an improved determination of the bright end of the luminosity function. These represent the most up-to-date luminosity functions for these redshifts at the time of this writing. The characteristic magnitude values from these studies are: $M^{*}=-20.98(z=4),-20.64(z=5),-20.24(z=6)$, $-20.14(z=7),-20.26(z=8)$, and $-18.3(z=10)$.

\section{OBSERVATIONS}

In this paper, we use a sample of high-redshift galaxies selected from HST data in the GOODS-S region. We review our methods below, and note that they are similar to those used in Finkelstein et al. (2012), thus the reader is referred there for further details.

\subsection{Data}

We use WFC3 imaging data from three separate programs. The first is the HUDF09 program (PID 11563; PI: Illingworth), which obtained deep imaging over the Hubble UltraDeep Field (HUDF) as well as its two flanking fields (which we will refer to as HUDF09-01 or PAR1 and HUDF09-02 or PAR2). The second data set comes from the WFC3 Early Release Science Program (ERS; PID 11359; PI: O'Connell; Windhorst et al. 2011), which imaged the northern $\sim 25 \%$ of the GOODS-S field. The third data set comes from the Cosmic Assembly Near-Infrared Deep Extragalactic Legacy Survey (CANDELS; PI: Faber \& Ferguson; Grogin et al. 2011; Koekemoer et al. 2011), which obtained deep imaging of the central $\sim 50 \%$ of GOODS-S (hereafter referred to as CANDELS DEEP), and less deep imaging of the southern $\sim 25 \%$ of GOODS-S (hereafter referred to as CANDELS WIDE; PID 12060, 12061, and 12062). We use the completed CANDELS data set in GOODS$\mathrm{S}$, which includes 10 epochs in the DEEP field, as well as both epochs of WIDE imaging. All three of these surveys obtained imaging in the F125W and F160W filters (hereafter $J_{125}$ and $\left.H_{160}\right)$. The CANDELS and HUDF09 surveys also obtained imaging in the F105W filter, while the ERS used the narrower F098M filter (hereafter $Y_{105}$ and $Y_{098}$, respectively). These fields and their associated depths are summarized in Table 1.

\subsection{Photometry and Sample Selection}

We performed photometry on all three data sets using the Source Extractor software (Bertin \& Arnouts 1996). We chose a single set of parameters that we applied to all images, which we tested to maximize detection of real sources while minimizing contamination (the key parameters are DETECT_MINAREA $=7$ and DETECT_THRESH $=0.6$ ). We did this in two-image mode, using a weighted sum of the $J_{125}$ and $H_{160}$ images as the detection image, and the three WFC3 bands, as well as the archival GOODS-S ACS F435W, F606W, $\mathrm{F} 775 \mathrm{~W}$, and F850LP (hereafter $B_{435}, V_{606}, i_{775}$, and $z_{850}$, respectively) bands as the measurement images. We also measured photometry on very deep F814W ACS imaging, obtained in parallel during the CANDELS observations. Recently obtained ACS imaging suffers from poor charge transfer efficiency; this can be corrected for detectable sources (Anderson \& Bedin 2010), but at this point it is unclear whether robust upper limits can be obtained from these data. We thus use the F814W imaging (which has had this correction applied) only as a veto band when inspecting $z=8$ galaxy candidates, as at these redshifts there should be no detectable flux in this band. All images were matched to the point-spread function (PSF) of the $H_{160}$ image prior to the photometry.

We selected our sample of high-redshift galaxies using the photometric redshift fitting software EAZY (Brammer et al. 2008). Rather than using the best-fit photometric redshift, we used the full probability distribution function (PDF) to select our galaxies. We do this by compiling samples in three redshift bins, $z=6,7$, and 8 . To make it into a given bin, an object must have a $\geqslant 3.5 \sigma$ detection in both the $J_{125}$ and $H_{160}$ bands, it must have $\geqslant 70 \%$ of its integrated PDF in the primary redshift solution, and it must have $\geqslant 25 \%$ of its integrated PDF within $z_{\text {sample }} \pm 0.8$ (i.e., for $z=6, \geqslant 25 \%$ of the integrated PDF must be at $5.2 \leqslant z \leqslant 6.8)$. Finally, objects which satisfy this criterion for multiple samples are placed in the sample which contains a larger fraction of the PDF (for this purpose, we also integrated the PDF for hypothetical $z=5$ and $z=9$ samples to prevent those objects from being placed in our $z=6$ and $z=8$ samples, respectively). These methods are similar to that used in Finkelstein et al. (2012), and their Figure 1 illustrates this selection technique.

This selection was performed separately for each imaging data set. All selected candidates were visually inspected, to screen against false detections such as diffraction spikes or oversplit regions on the edges of bright galaxies. Additionally, we screened against stellar contaminants, first by investigating 


\section{$z=6$}

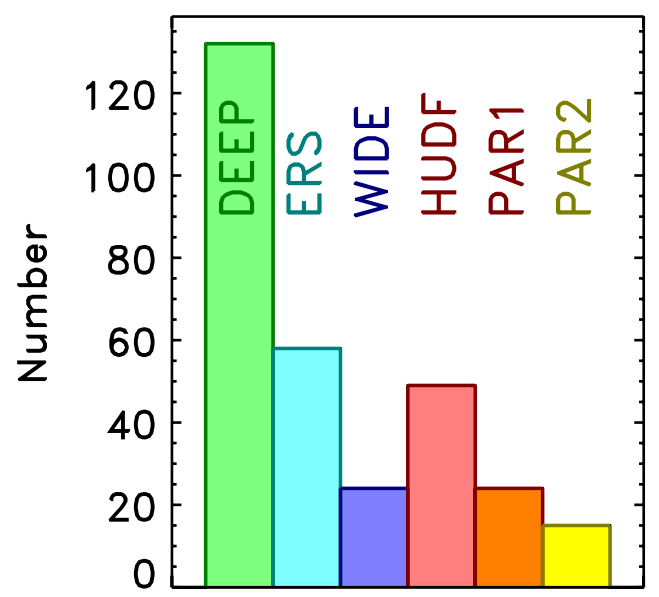

$z=7$

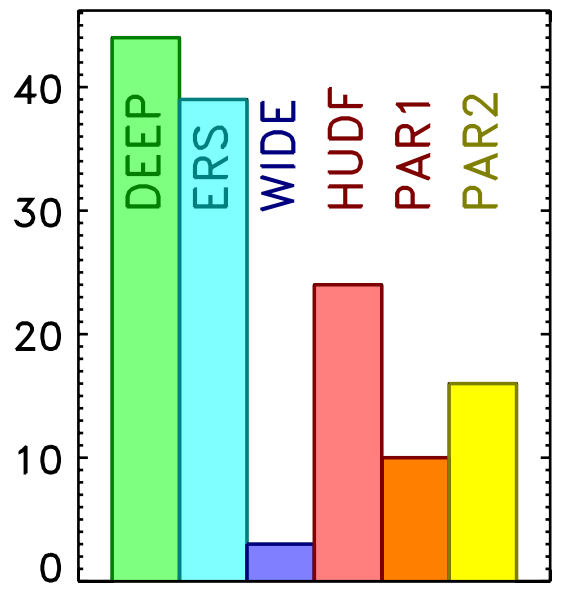

$z=8$

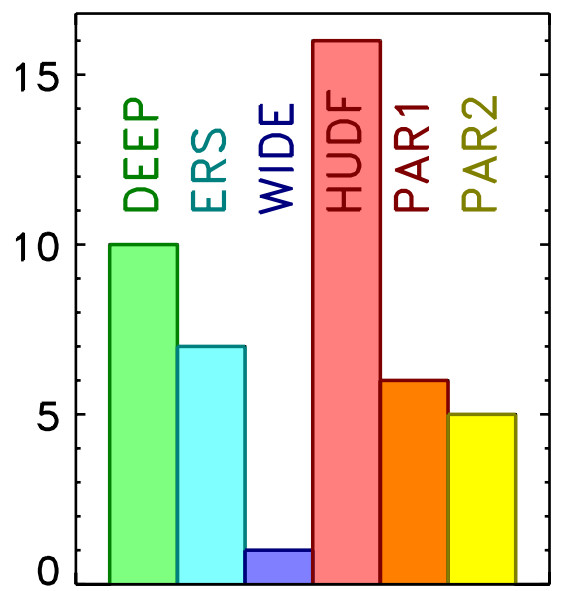

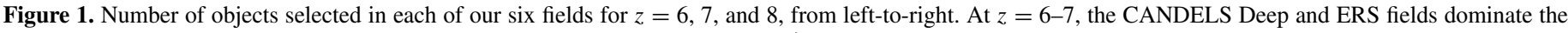

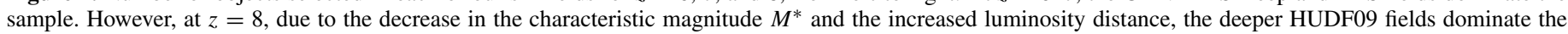
sample. The CANDELS Wide field contributes a non-negligible number of galaxies at $z=6$, but is not deep enough to contribute significantly at higher redshifts.

(A color version of this figure is available in the online journal.)

whether a given source is resolved, which we did by comparing the measured full width at half-maximum (FWHM) of the sources to that of stars in the image (where this measurement was performed by Source Extractor). For sources that had FWHMs close to that of stars in the image, we compared their colors to measured colors of late-types stars and brown dwarfs, integrating observed NASA Infrared Telescope Facility (IRTF) SpeX prism spectra through the ACS and WFC3 bandpasses. ${ }^{12}$ We found 15 likely M-dwarfs in our $z=6$ sample and 4 likely brown dwarfs in our $z=7$ sample. After clearing these contaminants from the sample, we had a total of 302,136 , and 45 galaxy candidates at $z=6,7$, and 8 , respectively. These samples are summarized in Figure 1, which highlights the importance of a wide area at $z=6$ and very deep imaging at $z=8$.

\section{ANALYSIS}

\subsection{Measuring the Rest-frame UV Light}

In order to study the contribution of the galaxies in our sample to the reionization of the universe, we need to measure their rest-frame UV absolute magnitudes. We have chosen to do this in the same manner as in Finkelstein et al. (2012), which we briefly summarize here. We first compute a grid of synthetic stellar population models over a range of redshifts, population ages, stellar metallicities, star formation histories, and dust attenuations, using the updated (2007) models of Bruzual \& Charlot (2003, hereafter BC03). We then found the best-fit model for a given galaxy in our sample via $\chi^{2}$ minimization, and then integrated this best-fit model spectrum through a $100 \AA$ wide square bandpass centered at rest-frame $1500 \AA$ to compute the flux at that wavelength. This was then converted to the absolute magnitude at $1500 \AA\left(M_{1500}\right)$ using the distance modulus to the best-fit photometric redshift. The uncertainty on $M_{1500}$ was derived via a series of 100 Monte Carlo simulations, where in each simulation each galaxy's fluxes were altered by a Gaussian random distribution with a mean of zero and a standard deviation equal to the photometric error for the band in question. In each simulation, a new redshift was chosen

\footnotetext{
12 This research has benefited from the SpeX Prism Spectral Libraries, maintained by Adam Burgasser at

http://pono.ucsd.edu/ adam/browndwarfs/spexprism.
}

based on the photometric redshift PDF for a given object; in this way the uncertainties on all derived parameters include the inherent uncertainty in the redshift. The $68 \%$ confidence range of $M_{1500}$ was then derived as the central $68 \%$ spread of $M_{1500}$ values from the simulations for each object.

\subsection{Incompleteness Correction}

Prior to computing the specific luminosity density, we first need to correct for incompleteness down to our limiting magnitude. We have done this by inserting mock galaxies into our images, running separate simulations for each redshift bin and each data set (for a total of 18 sets of simulations). In each simulation, we chose a uniform random distribution of redshifts ranging from $z_{\text {sample }} \pm 1$, as well as a range of $H_{160}$ magnitudes modeled after the number counts in a given field, but continuing the trend to fainter magnitudes. We then chose a stellar metallicity, population age, and color excess $E(B-V)$ from distributions which were tuned such that the recovered galaxies in a given simulation had a similar rest-frame UV spectral slope distribution as those of the recovered galaxy sample (ensuring that we are not correcting for, e.g., a population of red galaxies that do not exist in reality). A stellar population model was then created with these parameters and integrated through the bandpasses of interest to derive galaxy magnitudes in each band.

The image of each mock galaxy was created with the GALFIT software package (Peng et al. 2002). The position angle was drawn from a uniform random distribution, while the Sérsic index $n$ was drawn from a log-normal distribution, such that the mock sample was predominantly disk galaxies, with fewer bulge-dominated galaxies. Both the half-light radius and the axial ratio were tuned such that the recovered distributions matched the distributions from our observed galaxy sample (to prevent against, i.e., correcting for large galaxies that do not exist).

Samples of 100-200 mock galaxies were placed in subregions of the images in each field. The images were then treated in the same manner as in Section 2.2, in that we derived photometry with Source Extractor, and photometric redshifts were measured with EAZY. Samples were selected at each redshift using the same criteria as those used on our galaxy samples. This process was repeated until $\sim 10^{5}$ galaxies were 

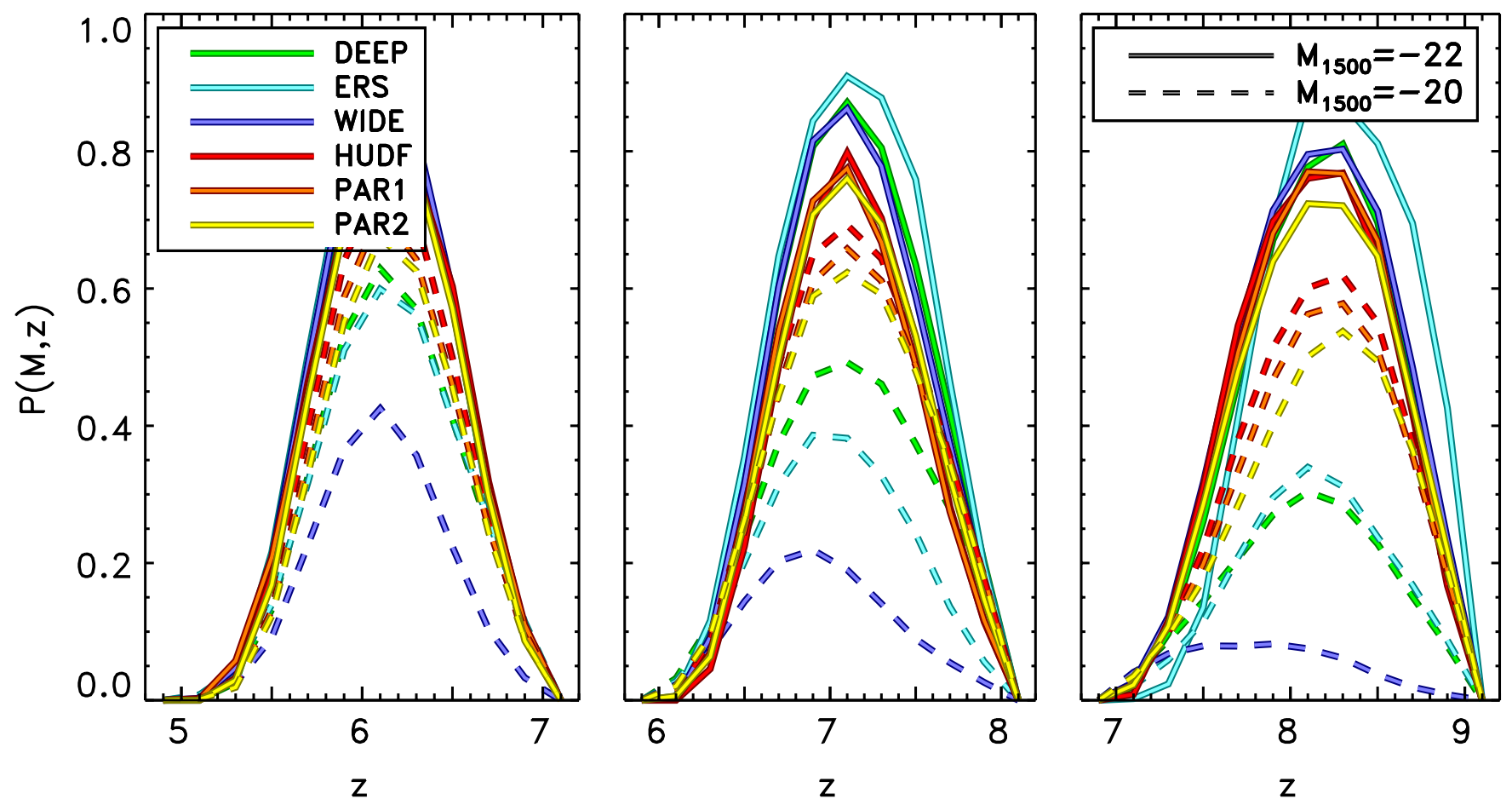

Figure 2. Summary of our completeness simulations. Each panel represents one of our redshift bins of $z=6,7$, and 8 from left-to-right. Each panel contains the probability of recovering a given galaxy (as a function of redshift and $M_{1500}$ ) for two magnitude values; $M_{1500}=-22$ (solid line) and $M_{1500}=-20$ (dashed line). At $z=6,7$, and $8, M_{1500}=-22$ corresponds to apparent UV magnitudes of 24.7, 24.9, and 25.1, and $M_{1500}=-20$ corresponds to 26.7, 26.9, and 27.1, respectively. While all fields can efficiently detect bright galaxies at any redshift, only the two HUDF09 fields can efficiently detect fainter galaxies at higher redshift, which is consistent with our recovered distribution shown in Figure 1. Although the HUDF09 fields are more efficient than the CANDELS Deep and ERS fields for fainter galaxies at $z=6$, the much larger area probed by the latter fields results in their dominating the sample at that redshift. We note that although they are of comparable area, the volume probed by the WIDE field is much less than the ERS. At fainter magnitudes, this is due to the increased depth in the ERS. However, even at bright magnitudes, the ERS is more complete at $z=7$ and 8, as the F098M filter used in the ERS does not overlap with F125W (while F105W, used in the remaining fields, does), allowing more precise photometric redshifts.

(A color version of this figure is available in the online journal.)

input and measured from the images (i.e., 500-1000 iterations). Typical numbers of recovered galaxies were $\sim 2-3 \times 10^{4}$ (depending on the field and redshift), where a galaxy is denoted as recovered if it is detected in the photometry and selected as a candidate in the redshift range of interest.

For each field and at each redshift, we then computed the probability that a given galaxy was recovered in our sample as a function of the input redshift and $M_{1500}$ (where here $M_{1500}$ was measured from the input spectrum to the simulations), defined as $\mathcal{P}(M, z)$. These values are shown at a few representative magnitudes in Figure 2.

\subsection{Evolution of the Rest-frame UV Specific Luminosity Density}

Using the value of $M_{1500}$ for each galaxy, as well as $\mathcal{P}(M, z)$ for each field and redshift, we computed the rest-frame UV specific luminosity density ( $\rho_{\mathrm{UV}}$ ), in units of $\mathrm{erg} \mathrm{s}^{-1} \mathrm{~Hz}^{-1} \mathrm{Mpc}^{-3}$ (specific refers to per unit frequency, while density refers to per unit volume). We converted the absolute magnitude $M_{1500}$ of each galaxy to the $1500 \AA$ A specific luminosity (i.e., $\mathrm{erg} \mathrm{s}^{-1} \mathrm{~Hz}^{-1}$ ) using the best-fit photometric redshift (while noting that the uncertainty in $M_{1500}$ includes the uncertainty in the redshift; see Section 3.1).

We computed the effective volume, which is the volume over which we are selecting galaxies given our imaging depths and selection criteria, defined as

$$
V_{\text {eff }}\left(M_{1500}\right)_{\text {field }}=A_{\text {field }} \int \frac{d V}{d z} \mathcal{P}\left(M_{1500}, z\right) d z,
$$

where $A_{\text {field }}$ is the area probed by a given field, which is given in Table 1 , and $d V / d z$ is the co-moving volume per square arcminute per redshift slice $d z$ at the redshift $z$. The value of $\rho_{\mathrm{UV}}$ is thus the sum of the specific luminosities divided by the effective volume for a given field and redshift, in each magnitude bin. We note that in the following, when we refer to measurements of our observed galaxy sample, or the observable galaxy sample at a given redshift, we refer to these incompleteness-corrected measurements.

We computed a total value of $\rho_{\mathrm{UV}}$ for each redshift, by first taking the sum of all specific luminosities in a given redshift sample in a given magnitude bin, and then dividing it by the sum of the effective volumes for each field in that magnitude bin. We corrected down to a limiting $M_{1500}=-18$, which corresponds to $0.13,0.14$, and $0.19 \times L^{*}$ at $z=6,7$, and 8 , respectively (as well as $0.06 \times L_{z=3}^{*}$; Steidel et al. 1999). The total value of $\rho_{\mathrm{UV}}$ was then the sum of these values over all magnitude bins. These values are shown as the large cyan circles in Figure 3 and are tabulated in Table 2.

The uncertainties quoted on $\rho_{\mathrm{UV}}$ are comprised of three components: Poisson noise, photometric scatter, and uncertainty in the effective volumes. These were included simultaneously in a series of $10^{4}$ bootstrap Monte Carlo simulations. In each simulation, we accounted for Poisson noise by selecting $N^{\prime}=N+A \times \sqrt{N}$ galaxies in each redshift bin through random sampling with replacement, where $N$ is the number of galaxies in the bin, and $A$ is a random number drawn from a normal distribution with a mean of zero and a standard 


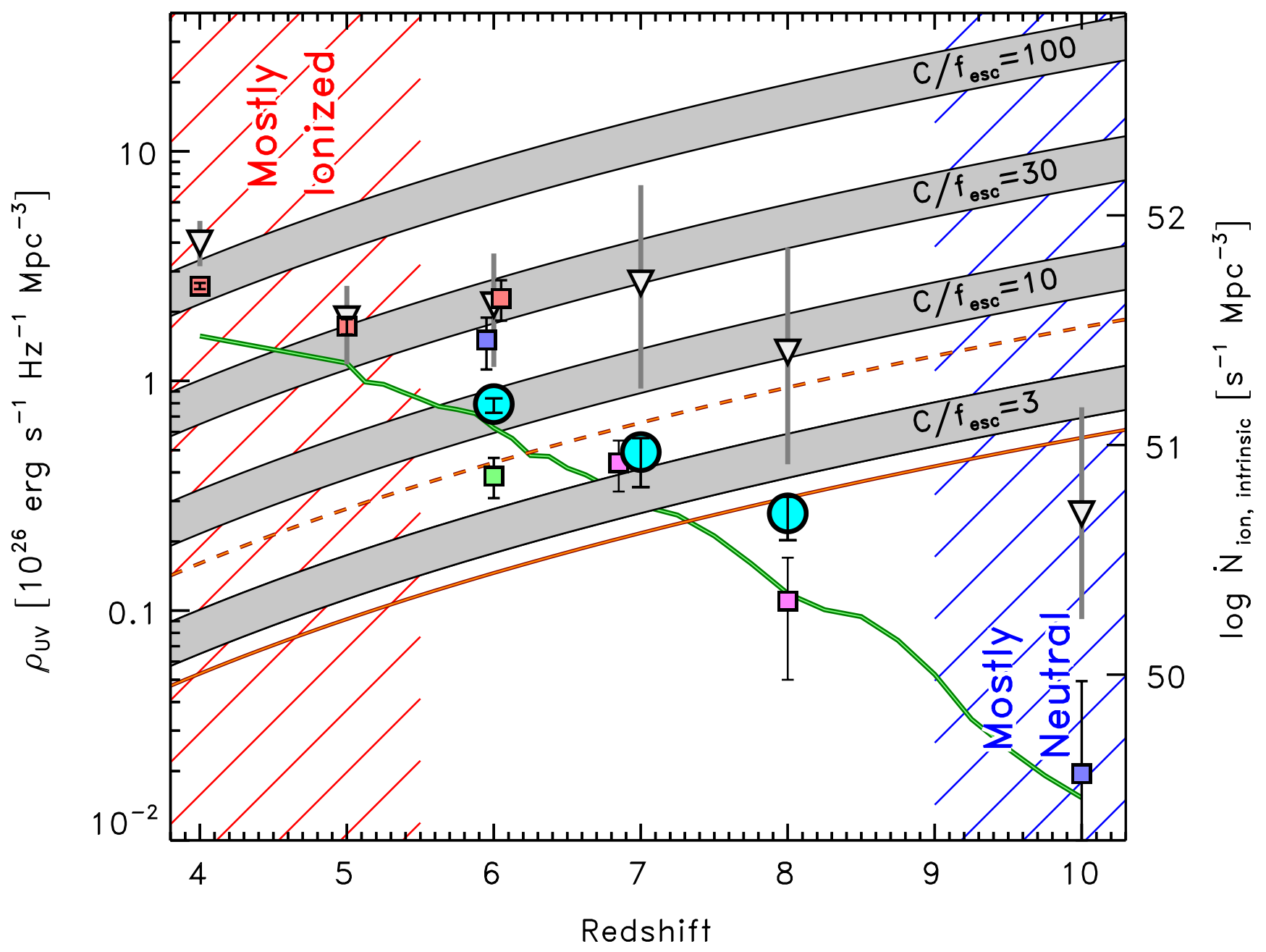

Figure 3. Specific luminosity density ( $\rho_{\mathrm{UV}}$ ) vs. redshift, where our observed samples, corrected for incompleteness down to $M_{1500}<-18$, are plotted as the cyan circles. Results from the literature are shown as squares at $z=4,5$, and 6 from the GOODS fields (red; Giavalisco et al. 2004), $z=6$ from the HUDF (green; Bunker et al. 2004), $z=7$ and 8 from the HUDF (purple; Finkelstein et al. 2010), $z=6$ from GOODS+HUDF, and $z=10$ from the HUDF (blue; Bouwens et al. 2006, 2011b). All squares have been adjusted to a limiting magnitude of -18 (with the exception of the $z=7$ and 8 points, which were not corrected for incompleteness). The inverted triangles denote the integrated luminosity functions down to $M_{\lim }=-13$ of Bouwens et al. (2007) at $z=4,5$, and 6 , Bouwens et al. (2011a) at $z=7$, Bradley et al. (2012) at $z=8$, and Bouwens et al. (2011b) at $z=10$, where the gray error bar denotes the uncertainty on these integrated values due to the uncertainty in the Schechter function parameters (particularly the faint-end slope $\alpha$ in the higher redshift bins). The wide gray curves denote the value of $\rho_{\mathrm{UV}}$ needed to sustain a fully reionized IGM at a given redshift, for a given ratio of the clumping factor $C$ over the escape fraction of ionizing photons $f_{\text {esc }}$ (Madau et al. 1999). The width of these curves represents changing stellar metallicities, from $0.02<Z / Z_{\odot}<1.0$. The orange curves show the critical value of $\rho_{\mathrm{UV}}$ from the models of Shull et al. (2012), the dashed line denotes an IMF with a slope of -2.35 and an upper-mass cutoff of $200 M_{\odot}$, while the solid line denotes an IMF slope of -1.9 with an upper-mass cutoff of $100 M_{\odot}$. The right-hand vertical axis shows the corresponding intrinsic number density (i.e., prior to escape) of ionizing photons for a given specific UV luminosity density, using the median of a range of ages and metallicities, and assuming a constant star formation history. This axis can be multiplied by the reader's choice of $f_{\text {esc }}$. The green curve shows the predicted luminosity density for sources brighter than $M_{1500}=-18$ from the hydrodynamic simulations of Finlator et al. (2011). (A color version of this figure is available in the online journal.)

Table 2

Rest-frame UV Specific Luminosity Density

\begin{tabular}{cccccccc}
\hline \hline$z$ & $N_{\text {Galaxies }}$ & $\begin{array}{c}\rho_{\mathrm{UV}} \\
M_{1500}<-18\end{array}$ & $\begin{array}{c}\rho_{\mathrm{UV}} \\
L>0.2 L^{*}\end{array}$ & $\begin{array}{c}\rho_{\mathrm{UV}} \\
L>0.5 L^{*}\end{array}$ & $\begin{array}{c}\rho_{\mathrm{UV}} \\
L>L^{*}\end{array}$ & $\begin{array}{c}V_{\text {eff }}\left(M_{1500}=-22\right) \\
\left(\mathrm{Mpc}^{3}\right)\end{array}$ & $\begin{array}{c}V_{\text {eff }}\left(M_{1500}=-20\right) \\
\left(\mathrm{Mpc}^{3}\right)\end{array}$ \\
\hline 6 & 302 & $0.79 \pm 0.06$ & $0.67 \pm 0.05$ & $0.44 \pm 0.04$ & $0.25 \pm 0.03$ & $3.07 \pm 0.06 \times 10^{5}$ & $2.28 \pm 0.03 \times 10^{5}$ \\
7 & 136 & $0.49 \pm 0.11$ & $0.43 \pm 0.06$ & $0.27 \pm 0.03$ & $0.17 \pm 0.02$ & $3.04 \pm 0.04 \times 10^{5}$ & $2.23 \pm 0.02 \times 10^{5}$ \\
8 & 45 & $0.27 \pm 0.06$ & $0.22 \pm 0.04$ & $0.08 \pm 0.02$ & $0.04 \pm 0.01$ & $2.96 \pm 0.05 \times 10^{5}$ & $1.94 \pm 0.02 \times 10^{5}$ \\
\hline
\end{tabular}

Notes. All specific luminosity density values are in the units of $10^{26} \mathrm{erg} \mathrm{s}^{-1} \mathrm{~Hz}^{-1} \mathrm{Mpc}^{-3}$. The effective volumes are quoted in comoving units and were derived from the completeness simulations discussed in Section 3.2. The uncertainties on the volumes come from bootstrap simulations, successively selecting $50 \%$ of the recovered simulated galaxies to assess the variation in $\mathcal{P}(M, z)$.

deviation of unity. We then account for the photometric noise by taking each of the galaxies in the modified sample and obtaining a new estimate, $M_{1500}^{\prime}$, for $M_{1500}$, where $M_{1500}^{\prime}$ is the original value $M_{1500}$ modified by a Gaussian with $\sigma$ equal to the photometric error (performed in flux space). Finally, we accounted for the uncertainty in the effective volume simulations by computing the uncertainty in $\mathcal{P}\left(M_{1500}, z\right)$ via a separate set of simulations, randomly selecting $N$ recovered simulated galaxies, 
where $N$ is $50 \%$ of the total number of recovered galaxies. This was done $10^{3}$ times, generating $10^{3} \mathcal{P}\left(M_{1500}, z\right)$ arrays. The uncertainty in $\mathcal{P}\left(M_{1500}, z\right)$ was derived as the standard deviation of the $10^{3}$ probability values at each magnitude and redshift. These uncertainties were then folded into our uncertainty on $\rho_{\mathrm{UV}}$ during the Monte Carlo simulations by modifying each value of the $\mathcal{P}\left(M_{1500}, z\right)$ array by a Gaussian with $\sigma$ equal to the uncertainty in $\mathcal{P}\left(M_{1500}, z\right)$. Thus, at each redshift, we obtained $10^{4}$ values for $\rho_{\mathrm{UV}}$ including the effects of sample size, photometric scatter, and uncertainty in the effective volumes. The uncertainty we quote is the central $68 \%$ range of these values.

We list the total effective volumes probed by our study at each redshift (at two representative magnitudes) in Table 2 . We note that these volumes are subject to our assumptions of the galaxy properties in our incompleteness simulations. As discussed in the previous subsection, the key parameters in recovering a galaxy are the color and size. While we have chosen what we believe are appropriate distributions of both quantities (see Section 3.2 for discussion), it is however interesting to examine how the effective volume would change if the assumptions were different. We have thus re-run our simulations at $z=$ 6 in each field, using a modified color and size distribution (in comparison to our default distributions, we broadened the distribution of dust attenuation and changed the size distribution to peak at smaller sizes, but with a higher large-radius tail). We found that the derived effective volumes from this modified simulation were $\sim 10 \%$ smaller than our nominal simulations in Section 3.2. Thus, the derived UV luminosity densities would be $\sim 10 \%$ higher if these volumes were more physically correct. However, as discussed above, we believe that our assumed physical property distributions are physically robust, so our effective volumes should be representative of the true volumes probed by our survey. As this test shows, if for whatever reason this is not the case, it creates a systematic uncertainty on $\rho_{\mathrm{UV}}$ on the order of $10 \%$ for modest changes in the input distributions.

\subsection{Critical Specific Luminosity Density to Sustain Reionization}

As shown in Figure 3, our results show a decrease with redshift in the specific luminosity density for the observable galaxy population down to $M_{1500}<-18$. In order to understand the implications of this trend on the reionization of the IGM, we need to connect our observable, $\rho_{\mathrm{UV}}$, to the necessary number of ionizing photons to sustain a fully reionized IGM. We do this in the same manner as in Finkelstein et al. (2010), where we followed Madau et al. (1999) to derive the required $1500 \AA$ luminosity density to sustain reionization:

$$
\begin{aligned}
\rho_{\mathrm{UV}}= & 1.24 \times 10^{25} \epsilon_{53}^{-1}\left(\frac{1+z}{8}\right)^{3}\left(\frac{\Omega_{b} h_{70}^{2}}{0.0461}\right)^{2} \\
& \times x_{\mathrm{H} \text { II }} \frac{C}{f_{\mathrm{esc}}} \mathrm{erg} \mathrm{s}^{-1} \mathrm{~Hz}^{-1} \mathrm{Mpc}^{-3}
\end{aligned}
$$

where $\Omega_{b}$ is the cosmic baryon density and $h_{70}$ is the Hubble parameter in units of $h=0.7$, and the constant 0.0461 is our assumed value of $\Omega_{b}\left(\times h_{70}^{2}\right)$ from WMAP 7 year data (Komatsu et al. 2011). The clumping factor of ionized hydrogen in the IGM is denoted by $C$ and ties in the average rate of recombinations in the IGM. The average escape fraction of ionizing photons from galaxies is denoted by $f_{\text {esc }}$. The variable $\epsilon_{53}$ is the number of Lyman continuum photons per unit of forming stellar mass in units of $10^{53}$ photons $\mathrm{s}^{-1}\left(M_{\odot} \mathrm{yr}^{-1}\right)^{-1}$. To determine this value, we used the updated $\mathrm{BC} 03$ models with a Salpeter initial mass function (IMF; with stellar masses from 0.1 to $100 M_{\odot}$ ) and a constant star formation history. We examined values of the stellar metallicity from 0.02 to $1.0 Z_{\odot}$, finding that $\epsilon_{53}$ ranges from 0.9 to $1.4 .{ }^{13}$ The volume ionized fraction is denoted by $x_{\mathrm{H} \text { II }}$. We start by assuming this is equal to unity and investigate changes in Section 4.6. The gray curves in Figure 3 denote the critical specific UV luminosity density from this equation required to sustain a fully reionized IGM at a given redshift, where the width of the curve denotes the range of $\epsilon_{53}$ values for differing metallicities. Should the IMF be more top heavy than Salpeter or the metallicities in these galaxies be $\ll 0.01 Z_{\odot}$, it would increase the number of ionizing photons per observed UV photon, making it easier for a given population to reionize the IGM (e.g., Chary 2008). We note that currently there is no strong evidence to support such very low metallicities of these galaxies (e.g., Bouwens et al. 2010; Finkelstein et al. 2010, 2012; Wilkins et al. 2011b; Finlator et al. 2011; Wise et al. 2012).

We reiterate that, under the assumption of $x_{\mathrm{HII}}=1$, these curves show the luminosity density required to maintain a fully ionized IGM. Thus, if at a given redshift our galaxies do not reach this threshold, it may not mean that sources of ionizing photons are missing, it may just indicate the epoch when the neutral fraction is no longer negligible. As the ionized fraction falls, one can calculate the luminosity density necessary to maintain a given volume ionized fraction by lowering the value of $x_{\mathrm{H}}$ II Previous observations constrain $x_{\mathrm{H}}$ to equal unity at $z<6$ (e.g., Fan et al. 2006; though see, e.g., Mesinger 2010; McGreer et al. 2011), and it is likely negligible by $z>15$ (cf. Section 4.6). As we are just pushing back into the reionization epoch, we assume this parameter equals unity, but we note that by $z \sim 8$ it may be substantially reduced (possibly to $\sim 0.5-0.7$, e.g., Bolton \& Haehnelt 2007; Pritchard et al. 2010; Bolton et al. 2011; Mortlock et al. 2011; Pentericci et al. 2011). The primary effect of this is that a lower ionized volume fraction makes it easier for galaxies to keep the ionized portion of the IGM in an ionized state. The reader can take this into account when investigating the various gray curves in Figure 3.

\section{DISCUSSION}

\subsection{Assumptions}

The critical curves for sustaining a fully reionized IGM are shown as the gray bands in Figure 3, for different assumptions of the ratio between the clumping factor and escape fraction of ionizing photons, $C / f_{\text {esc }}$. The clumping factor of ionized hydrogen in the IGM is derived theoretically, and up until recently, had been thought to be very large, $C \sim 30$ (e.g., Gnedin \& Ostriker 1997). This high value necessitates more ionizing photons to reionize a given volume, leading a number of studies to conclude that galaxies could not reionize the universe (e.g., Bunker et al. 2004). However, these early simulations included all gas in their computation of the clumping factor. It may be more accurate to only consider gas that is in the IGM, as absorption by gas in the ISM will be accounted for in the escape fraction. Due to a better understanding of the interface between the IGM and the circumgalactic medium, and also because of an improved understanding of the feedback

\footnotetext{
13 These values assumed an age of 50 Myr. For a constant star formation
} history, $\epsilon_{53}$ is invariant for ages larger than $10 \mathrm{Myr}$. 
from reionization (e.g., Miralda-Escudé et al. 2000; Pawlik et al. 2009; Shull et al. 2012) (see also Wise \& Abel 2005; Iliev et al. 2006; Raičević \& Theuns 2011) and improvements in our understanding of self-shielding (e.g., McQuinn et al. 2011), recent simulations yield much lower values of $C \sim 1-5$ (see also Faucher-Giguère et al. 2008). Recent simulations by Finlator et al. (2012) show that when averaging only over gas that is sufficiently underdense that its ionization state is determined by the global UV background rather than by the next nearest galaxy (the impact of circumgalactic gas being more appropriately attributed to $f_{\text {esc }}$ ), $C \sim 3$ at $z=6$, dropping to $\sim 2$ by $z=8$.

The escape fraction of ionizing photons from galaxies is also poorly understood and is one of the primary goals of a number of observational studies. This is impossible to directly observe at the redshifts of our study, as any escaping ionizing photons will be absorbed by lingering neutral gas in the IGM. Direct detection is possible at $z<4$, but the results have been mixed. Siana et al. (2010) searched for ionizing photons with UV imaging of $z \sim 1$ star-forming galaxies, finding only nondetections, resulting in an upper limit on the relative escape fraction of ionizing photons to UV photons of $f_{\text {esc,rel }}<2 \%$ (see also Siana et al. 2007). At $z \sim 3$, Shapley et al. (2006) found that 2/14 LBGs have relative escape fractions of $\sim 50 \%-100 \%$, with the remainder having $f_{\text {esc, rel }}<25 \%$. A number of other studies have detected escaping ionizing photons at $z \sim 3-4$ (e.g., Steidel et al. 2001; Iwata et al. 2009; Vanzella et al. 2010; Nestor et al. 2011, though we note some of these studies observed in the same field (SSA22), thus they may have sources in common), though space-based imaging may be necessary to exclude contamination from intervening sources (e.g., Vanzella et al. 2012), and an examination of the photoionization rate in the Lyman alpha forest implies $f_{\text {esc }}<1 \%$ at $z=3$ (FaucherGiguère et al. 2008).

Thus, it appears is if the average escape fraction of ionizing photons may be increasing with increasing redshift (or, alternatively, the fraction of galaxies exhibiting $f_{\mathrm{esc}}$ significantly larger than zero rises with redshift). This is supported by the analysis of Inoue et al. (2006), who combined non-detections of escaping ionizing photons at $z<3$ with the ionizing background intensities derived at higher redshift to conclude that $f_{\text {esc }}$ rises from $<0.01$ at $z=1$ to $\sim 0.1$ at $z=4$. Future results from UV observations from CANDELS as well as in the HUDF (PI: Teplitz) will shed more light on this issue, but there are a few observational clues to support this interpretation. A recent study by Kuhlen \& Faucher-Giguère (2012) have combined all observational evidence (luminosity functions, WMAP, $\mathrm{kSZ}$, and Ly $\alpha$ forest measurements) to create concordance models of reionization, which implies an increasing escape fraction at higher redshift with $f_{\text {esc }}$ higher by perhaps as much as $10 \times$ at $z>4$. Additionally, the fraction of galaxies exhibiting $\mathrm{Ly} \alpha$ emission (with a rest-frame Ly $\alpha$ equivalent width $>20 \AA$ ) rises with redshift, from $\sim 25 \%$ at $z \sim 3$ (e.g., Shapley et al. 2003) to $\gtrsim 60 \%$ at $z \sim 6$ (e.g., Stark et al. 2010, 2011). Whatever physical mechanism allows a larger fraction of Ly $\alpha$ photons to escape will likely also allow a larger fraction of ionizing photons to escape (to a point; if all ionizing photons escape, no Ly $\alpha$ will be produced). One possible scenario is that the covering fraction of neutral gas decreases with increasing redshift. Observational evidence for this has been found, as Jones et al. (2011) measured that the low ionization absorption lines in a stacked rest-frame UV spectrum of $z=4$ galaxies were weaker than at $z=3$, implying a lower covering fraction of neutral gas at higher redshift.
Additionally, they stacked galaxies with and without Ly $\alpha$ emission, and found weaker absorption features in the stack of galaxies with Ly $\alpha$ emission. This supports a scenario where galaxies at higher redshift have a lower neutral gas covering fraction, allowing both more ionizing and Ly $\alpha$ photons to escape directly to the observer, and that the observed increase in the incidence at Ly $\alpha$ emission at higher redshift may also trace an increase in the ionizing escape fraction.

The reduced covering fraction may be due to star formation activity. The specific star formation rate (sSFR; star-formation rate per unit mass) is remarkably constant from $z \sim 4$ to 7 (e.g., Stark et al. 2009; González et al. 2010). However, the typical galaxy physical size at a given luminosity decreases toward higher redshift (e.g., Ferguson et al. 2004; Oesch et al. 2010a; Ryan et al. 2012), thus the constant sSFR is occurring over a smaller surface area, which can result in the star formation episode driving gas from the galaxies more easily (e.g., Kornei et al. 2012). In addition, the stellar masses of galaxies have been observed to decrease with increasing redshift at a fixed fraction of $L^{*}$, reducing the gravitational potential, and also allowing more efficient winds (e.g., Stark et al. 2009; Finkelstein et al. 2010; González et al. 2010; Labbé et al. 2010; McLure et al. 2011), plausibly decreasing the gas covering fraction, allowing a higher escape fraction. We note however that these measurements solely constrain the stellar masses, not the total baryonic mass, which may not evolve as strongly. Finally, a higher escape fraction is also consistent with the observed galaxy luminosity density and the photoionization rate inferred from observations of the Ly $\alpha$ forest in $z \geqslant 6$ quasars, which imply $f_{\text {esc }} \sim 20 \%-30 \%$ at $z \sim 6$ (see Section 4.5; Bolton \& Haehnelt 2007).

A number of theoretical studies have examined the escape of ionizing photons, both as a function of redshift and halo mass (e.g., Ricotti \& Shull 2000; Fernandez \& Shull 2011; Gnedin et al. 2008; Wise \& Cen 2009; Yajima et al. 2011; Haardt \& Madau 2012; Conroy \& Kratter 2012). For example, Yajima et al. (2011) investigated this quantity from $3<z<6$. At $z=6$, they found that the escape fraction was $>10 \%$ for all halos more massive than $10^{11} M_{\odot}$ (which likely encompasses the majority of the galaxies in our sample), with $f_{\text {esc }}$ rising toward lower halo masses, for an overall average of $40 \%$. Razoumov \& Sommer-Larsen (2010) investigate this at higher redshifts and found that typical escape fractions in halos with masses from $10^{10}$ to $10^{11} M_{\odot}$ at $z=6-8$ are $\sim 50 \%-80 \%$. The radiative transfer simulations of Haardt \& Madau (2012) also find that a relatively high escape fraction, and one which rises with redshift from $10 \%$ to $30 \%$ from $z=6 \rightarrow 8$, is necessary to match the emissivity measurements from the Ly $\alpha$ forest. Another recent study, Conroy \& Kratter (2012) investigated the impact of runaway massive stars on the overall escape fraction. They found that by accounting for the motions of these stars the escape fractions in lower mass galaxies could rise, as the smaller sizes of lower mass galaxies would allow massive stars to emerge from the gas disk prior to exploding. They found that galaxies with halo masses less than $10^{10} M_{\odot}$ have escape fractions enhanced 3-6× over models without runaway stars. Various other models find that the escape fraction varies (either higher or lower) with halo mass due to a variety of effects (e.g., Gnedin et al. 2008; Wise \& Cen 2009; Fernandez \& Shull 2011), including holes in the ISM from supernovae (e.g., Yajima et al. 2009). Thus, a relatively high escape fraction and an escape fraction that varies with halo mass and redshift appears justified, though it remains to be verified observationally. 
In the following discussion, we will use the value of $C / f_{\text {esc }}=$ 10 as our canonical model, which represents a scenario of $C=$ 3 and $f_{\text {esc }}=30 \%$, consistent with the above results. Should the reader prefer different values, we show in Figure 3 critical curves for sustaining a fully reionized IGM for four values of $C / f_{\text {esc }}: 3,10,30$, and 100 . In our discussion, we will discuss the impact of a different escape fraction on our conclusions.

\subsection{Can the Observed Galaxies Sustain an Ionized IGM?}

In Figure 3, we show three previous results at $z=6$, all derived solely using ACS optical imaging. The first, from Giavalisco et al. (2004), is based on a sample of $117 \mathrm{i}^{\prime}$-dropout galaxies in the two GOODS fields. Correcting their sample for incompleteness down to $M_{\mathrm{UV}}=-19.3$, they found $\rho_{\mathrm{UV}}=$ $1.15 \pm 0.23 \times 10^{26} \mathrm{erg} \mathrm{s}^{-1} \mathrm{~Hz}^{-1} \mathrm{Mpc}^{-3}$. To convert this to our common magnitude limit of $M_{\mathrm{UV}}=-18$, we use the $z=6$ luminosity function of Bouwens et al. (2008), finding a correction factor of 2.0 (i.e., $\rho_{\mathrm{UV}}[M<-18]=2.3 \pm 0.46 \times$ $10^{26} \mathrm{erg} \mathrm{s}^{-1} \mathrm{~Hz}^{-1} \mathrm{Mpc}^{-3}$ ). We note that in their study, the existing depth in the $z^{\prime}$ data was 26.5; the data used in our present study are a full magnitude deeper.

The second observation, from Bunker et al. (2004), is based on a sample of $54 \mathrm{i}^{\prime}$-dropout galaxies in the HUDF, finding $\rho_{\mathrm{UV}}[M<-18.2]=0.357 \pm 0.071 \times 10^{26} \mathrm{erg} \mathrm{s}^{-1} \mathrm{~Hz}^{-1} \mathrm{Mpc}^{-3}$; we apply a factor of 1.08 to convert this to our common magnitude limit. Finally, the third study, from Bouwens et al. (2006), uses both of the aforementioned data sets, including also the HUDF parallel fields, for a total sample of $\sim 500$ galaxies, finding $\rho_{\mathrm{UV}}[M<-17.5]=1.77 \pm 0.45 \times$ $10^{26} \mathrm{erg} \mathrm{s}^{-1} \mathrm{~Hz}^{-1} \mathrm{Mpc}^{-3}$; we apply a correction factor of 0.85 to these observations to convert to $\rho_{\mathrm{UV}}[M<-18]$.

Interestingly, while the Bunker et al. (2004) survey goes deeper, down to $M_{\mathrm{UV}} \sim-18.2$, than the shallower GOODS data from Giavalisco et al. (2004), one would have expected the former to yield a larger value of $\rho_{\mathrm{UV}}$ (prior to the correction), but the opposite is observed. After we correct all three studies to a common magnitude limit, we would expect them to be in agreement, which is not the case, as they differ by more than a factor of five. There are a few reasons why such a discrepancy is not surprising. The first is due to cosmic variance, as the Bunker et al. (2004) study only probed the $11 \mathrm{arcmin}^{2}$ ACS data in the HUDF, while the Giavalisco et al. (2004) and Bouwens et al. (2006) studies included the $\sim 300 \mathrm{arcmin}^{2}$ GOODS fields. Second, as these galaxies were selected only with ACS imaging in all three surveys, they were observed in only a single band $\left(z^{\prime}\right)$. This can be dangerous, as the likelihood of selecting a noise peak is greatly enhanced when using a single band detection versus dual band (see Bouwens et al. 2011b and Yan et al. 2010 for a similar discrepancy in single-band-selected $z=10$ galaxy number counts, though the $z=10$ disconnect is greatly enhanced due to the extreme faintness of such sources).

The time is thus ripe to re-investigate the luminosity density at $z=6$, using our up-to-date data set. Our current sample of $z=6$ galaxies is more robust, as our selection over both the GOODS-S field and the HUDF09 fields covers both a large volume and a large dynamic range in object brightness. Additionally, the availability of the WFC3 data allows us to observe $z=6$ candidate galaxies in four bands, increasing the robustness of the sample. In particular, observations at four wavelengths throughout the rest-frame UV yield much more robust estimates of $M_{\mathrm{UV}}$ than the single point previously available at $z=6$. We find $302 z=6$ galaxy candidates, or nearly double the size of the Giavalisco et al. (2004) and Bunker et al. (2004) samples combined (though less than Bouwens et al. 2006; we expect this to be rectified when we soon incorporate the incoming CANDELS data in the GOODS-N field). We measure $\rho_{\mathrm{UV}}=$ $0.79 \pm 0.06 \times 10^{26} \mathrm{erg} \mathrm{s}^{-1} \mathrm{~Hz}^{-1} \mathrm{Mpc}^{-3}$, which is in between the previously measured values discussed above, with a much smaller uncertainty.

As shown in Figure 3, the observable (i.e., $M_{1500}<-18$ ) $z=6$ galaxy population is capable of sustaining reionization for our canonical model of a low clumping factor and a moderate average escape fraction $\left(C=3\right.$ and $\left.f_{\text {esc }}=30 \%\right)$. Thus, in a change from a number of previous studies (e.g., Yan \& Windhorst 2004; Bunker et al. 2004), it is not required to invoke galaxies fainter than our detection threshold to sustain a fully reionized IGM by $z=6$, due to both our more robust measurement as well as the updated assumptions about the clumping factor (see also Kuhlen \& Faucher-Giguère 2012). Nor do we need to invoke an escape fraction of unity, as $f_{\text {esc }} \sim 30 \%$ on average is sufficient (if $C \approx 3$ ). Finally, we reiterate that this is also independent of assumptions about the form and faint-end cutoff of the luminosity function. We note that our inclusion of deeper $z^{\prime}$ imaging, multiple fields, as well as near-infrared imaging yields a more robust estimate of the luminosity density, in contrast to the previous observations, which differed by a factor of $>5 \times$.

Different conclusions are reached at $z=7-8$. As shown in Figure 3, the specific UV luminosity density experiences a steady decline with redshift from $z=4$ to 8 . Combined with the denser IGM at higher redshift, necessitating a higher $\rho_{\mathrm{UV}}$ to compensate for the shorter recombination time, the observable galaxy population at $z=7-8$ falls short of what is needed to sustain a fully reionized IGM, unless the escape fraction is unity (which is unlikely to be the case for all galaxies). A similar result is seen in the simulations of Salvaterra et al. (2011), as shown in their Figure 8. They find that galaxies observable with $H S T$ can sustain a reionized IGM if $f_{\text {esc }}=20 \%$ and $C=5$ at $z=6$, with $C<2$ (or a higher escape fraction) necessary at $z \geqslant 7$ to maintain reionization with the observable galaxies. However, their simulation also probes down to $M_{\mathrm{UV}}=-15$, and at that level they find that the observable galaxies with $H S T$ only account for $\sim 20 \%$ of the total number of escaping ionizing photons.

At $z=7$, we find that the observed galaxies only account for $\sim 50 \%$ of the required UV luminosity density inferred to sustain reionization. As we discuss later in Section 4.7, observations reaching $\sim 1$ mag fainter may yield enough galaxies (assuming the published luminosity functions) to account for a fully reionized IGM if $C / f_{\text {esc }}=10$, similar to conclusions at this redshift from Robertson et al. (2010). We explore the possible contribution from galaxies below our detection threshold in Section 4.4 and examine possible changing escape fractions in Section 4.5.

Our observations from only the observed galaxy population are consistent with independent evidence that the universe was not completely reionized at $z \geqslant 7$. Studies of both Ly $\alpha$ emission (e.g., Stark et al. 2010, 2011) and quasar near-zones (e.g., Fan et al. 2006) at $z \sim 6$ yield results consistent with a negligible neutral fraction, implying that reionization was complete by $z=6$. However, recent spectroscopic follow-up of $z \sim 7$ galaxies has yielded a much lower success rate of Ly $\alpha$ emission detection than there should have been given the Ly $\alpha$ EW distributions at $z=4-6$ (Stark et al. 2010; Fontana et al. 2010; Pentericci et al. 2011; Ono et al. 2012; Schenker et al. 2012; Treu et al. 2012), implying neutral fractions as high as $\sim 50 \%$. 
A similar decrease in the Ly $\alpha$ luminosity function has been measured from $z=5.7$ to $z=6.6$ (e.g., Ouchi et al. 2010; $\mathrm{Hu}$ et al. 2010), though see also Malhotra \& Rhoads (2004). Follow-up high-resolution spectroscopic study of the near-zone around the recently discovered $z=7$ quasar ULAS J1120+0641 (Mortlock et al. 2011) yields evidence for a neutral fraction $>10 \%$. We note that the Ly $\alpha$ results are very preliminary and are also dependent on the neutral gas distribution within the galaxy (e.g., see Section 6.3 of Finkelstein et al. 2012), and the quasar results are based on a single sight line, which could be biased by a nearby cloud of neutral gas (e.g., Bolton et al. 2011), thus more data are needed to conclusively show that the neutral fraction is indeed rising. In any case, if our assumptions of a low clumping factor and a moderate escape fraction are reasonably correct, then the combination of galaxy, quasar, and spectroscopic observations points to an end to reionization by $z=6$, but not earlier than $z \sim 6.5$. This matches well with the conclusions we make solely from the observed galaxies, though it is likely that galaxies fainter than our detection limit are playing a strong role (though this depends on the limiting magnitude and whether the escape fraction is luminosity dependent). This then implies that through some combination of limiting magnitude and luminosity-dependent escape fractions, the observed galaxy populations are an excellent tracer of the ionizing photon background.

In order to place these results in context, we examine the recent study of Kuhlen \& Faucher-Giguère (2012), who combined the WMAP and SPT results with the observed luminosity function evolution (extrapolated to higher redshift), and $z<6$ Ly $\alpha$ forest measurements to investigate concordance models of reionization. They find that the available data predict that the universe was $\sim 50 \%$ ionized at $z \sim 10$ when using the conservative constraint from the SPT measurements on the duration of reionization (Zahn et al. 2012), as recent work by Mesinger et al. (2012) shows that there likely do exist correlations between the thermal SZ power and the cosmic infrared background; in the presence of these correlations, the constraint on the duration of reionization degrades to $\Delta z<7.9$ (Zahn et al. 2012; Mesinger et al. 2012). In the presence of this constraint, Kuhlen $\&$ Faucher-Giguère (2012) find that scenarios invoking a very high contribution from very faint galaxies will result in a reionization history which is too extended. As shown in Figure 3, if the IGM is truly $\sim 50 \%$ ionized at $z \sim 10$, then there will need to be a significant contribution from faint galaxies, necessitating a longer duration of reionization.

We note that our quoted rest-UV luminosities are not corrected for dust attenuation. In Finkelstein et al. (2012), we examined the average dust attenuation as a function of redshift, finding that $z=6$ galaxies appeared to contain little dust, and $z=7$ galaxies appeared to be dust free (due to only two detected fluxes, robust estimates of the extinction at $z=8$ were difficult, though they were consistent with little-to-no dust). Using the values of the UV spectral slope $\beta$ from that work, and the conversion from $\beta$ to the attenuation at $1600 \AA$ from Meurer et al. (1999 see discussion in Finkelstein et al. 2012 for the applicability of this relation at high redshift), we find that $A_{1600}=$ $0.31 \mathrm{mag}$ at $z=6$, and $A_{1600}=0 \mathrm{mag}$ at $z=7$ and 8. Thus, accounting for dust does not change the above conclusions at $z=7$ and 8 , while the observed specific luminosity density may in fact be up to $30 \%$ higher at $z=6$. However, any dust present will likely also attenuate the ionizing photons, though the dust attenuation curves are not well sampled at such blue wavelengths (e.g., Calzetti et al. 1994). In light of this, we con- clude that using the observed luminosity density is appropriate, assuming that the ionizing radiation is not significantly more (or less) attenuated than the rest-frame $1500 \AA$ light.

In Figure 3, we also show (in orange) critical values of $\rho_{\mathrm{UV}}$ from the reionization models of Shull et al. (2012) for a varying IMF (see their Equation 9). The calculation to derive these values is similar to that of Madau et al. (1999), except that these models allow an explicit IGM temperature dependence. Shull et al. (2012) also provide a convenient method to determine $\epsilon_{53}$ (which they refer to as $Q_{L y C}$ ) for a wider variety of stellar population parameters, including a varying IMF. Thus, it is interesting to examine this model for a few representative parameter values to see if it affects our earlier conclusions.

We first used the models of Shull et al. (2012) to derive the critical luminosity density assuming the same parameters as our default models from Madau et al. (1999), namely, $C / f_{\text {esc }}=10$, a Salpeter IMF, and a stellar metallicity $20 \%$ of solar. We also needed to assume a value for the diffuse IGM temperature; Shull et al. (2012) suggest a value of $10^{4} \mathrm{~K}$, as simulations suggest $5000 \mathrm{~K}<\mathrm{T}<20000 \mathrm{~K}$, consistent with temperatures observed in Ly $\alpha$ forest measurements of the IGM (e.g., Davé et al. 2001; Becker et al. 2007). The critical luminosity density from this relation is consistent with the $C / f_{\text {esc }}=10$ curve already in Figure 3 , though we note that increasing or decreasing the temperature can significantly raise or lower the derived critical value.

To investigate the impact of a changing IMF, we use the recent empirical results from Finkelstein et al. (2011), who found evidence for a top-heavy IMF and/or a $\sim 200 M_{\odot}$ upper mass limit based on an elevated ratio of Paschen $\alpha$ to the farinfrared emission in a lensed submillimeter galaxy at $z=2.5$. If we first keep the IMF slope fixed to -2.35 and change the upper mass end to $200 M_{\odot}$ (up from $100 M_{\odot}$ ), we derive a critical luminosity density that changes only slightly from the nominal case (slightly below the $C / f_{\text {esc }}=10$ curve). If however we keep the upper mass end limit at $100 M_{\odot}$ and change the slope of the IMF to -1.9 , which was the best-fit value from Finkelstein et al. (2011), then we find a vastly different result, shown as the solid orange curve in Figure 3. If the IMF is in fact significantly topheavy at high redshift, then as expected the critical luminosity density for sustaining reionization decreases, allowing even our observed sample of galaxies at $z=7$ to sustain an ionized IGM with an average escape fraction of only $30 \%$.

Finally, in Figure 3 we show in green the predicted luminosity density for galaxies at $M_{1500}<-18$, derived from a radiation hydrodynamic simulation that incorporates both galactic outflows and a self-consistently grown ionizing background as in Finlator et al. (2011), while subtending a $12 h^{-1}$ Mpc volume at the same mass and spatial resolution. At all redshifts, these predicted values fall short of our observed luminosity densities. Some of this discrepancy may be due to volume limitations, but as we show below, the sub- $L^{*}$ galaxies are the dominant contributors, hence they should be reasonably well represented. This discrepancy could indicate that a higher star formation efficiency in the models is warranted, perhaps via weaker outflows. It also relaxes the requirements for the ionizing escape fraction. In particular, the Finlator et al. (2011) models required an escape fraction of $50 \%$ in order to complete reionization by $z=6$; weakening feedback by a factor of 2-3 would suppress the required escape fraction by the same factor, bringing it closer to the observed values at lower redshift (Shapley et al. 2006; Siana et al. 2010; Nestor et al. 2011), as well as those we find below in Section 4.5. 


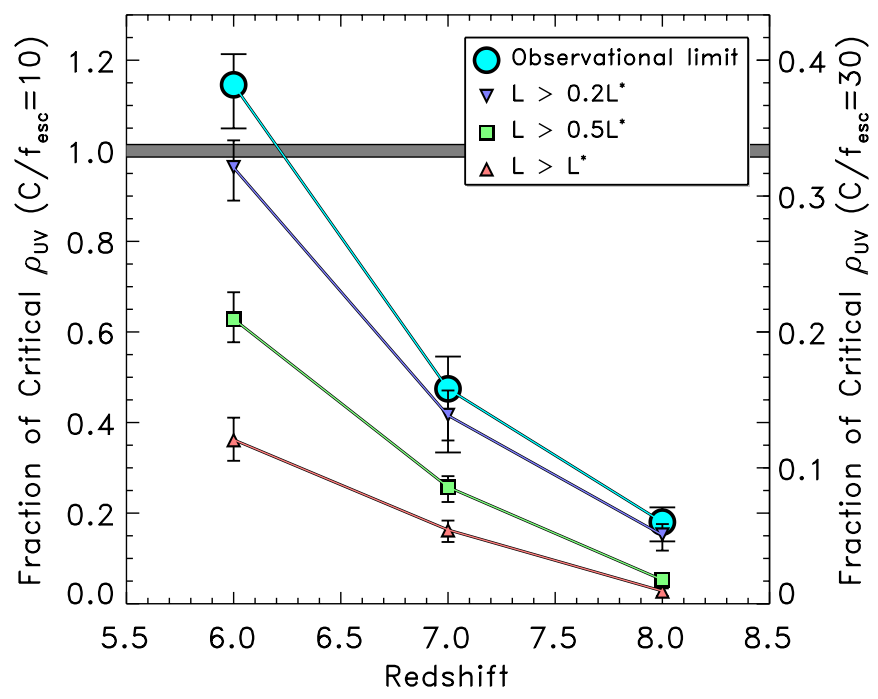

Figure 4. Fraction of the critical specific UV luminosity density necessary for sustaining reionization (denoted by the gray bar for $C / f_{\text {esc }}=10$ ) obtained from the (incompleteness-corrected) observed galaxy population (large cyan points) as a function of redshift, assuming $C / f_{\text {esc }}=10$ (left vertical axis) and 30 (right vertical axis). The blue inverted triangles, green squares, and red triangles show the critical fraction for galaxies with luminosities greater than $0.2,0.5$, and $1 \times$ the characteristic luminosity $L^{*}$, respectively. At $z=6$, where the observed population can reionize the universe, this figure highlights that while galaxies below our detection limit are not necessary, it is the fainter portion of the observed population which is dominating $\rho_{\mathrm{UV}}$, as galaxies with luminosities greater than $0.2 L^{*}$ contribute $\sim 2.7 \times$ more than $L>L^{*}$ galaxies. This is even more so in the case at $z=7$ and 8 , though at these redshifts the entire observed population is short by a factor of $\sim 2.5-3$ of the ionizing photons necessary to sustain reionization for $C / f_{\text {esc }}=10$.

(A color version of this figure is available in the online journal.)

\subsection{Contribution of Different Luminosity Ranges}

We now investigate how the different luminosity ranges of our observed galaxy sample contribute to the total specific UV luminosity density. We reiterate that this is independent of any luminosity function parameterization (with the exception of the definition of the characteristic luminosity $L^{*}$ ). In Figure 4, we show the fraction of the critical value of $\rho_{\mathrm{UV}}$ necessary for sustaining a fully reionized IGM reached by our observed population at each redshift, for all galaxies (large circles), and for galaxies with luminosities greater than $0.2,0.5$, and $1 \times L^{*}$ (small colored symbols), where the appropriate value of $L^{*}$ for each redshift is used.

At $z=6$, this figure reinforces our observation that the observable population at $z=6$ appears sufficient to maintain an ionized IGM, while those at $z=7$ and 8 are not. At $z=6$, the ratio of the specific UV luminosity density from galaxies with $L>0.2 L^{*}$ to that of galaxies with $L>L^{*}$ is a factor of $\sim 2.7$. This highlights that it is very much the sub- $L^{*}$ galaxies which are dominating the reionization photon budget. Additionally, galaxies with $0.2 L^{*}<L<0.5 L^{*}$ contribute a roughly equal amount to reionization as those with $L>L^{*}$. These ratios are similar at $z=7$.

At $z=8$, the results are even more tilted in favor of the fainter galaxies, with the ratio of $\rho_{\mathrm{UV}}$ from galaxies with $L>0.2 L^{*}$ to that of galaxies with $L>L^{*}$ at a factor of $\sim 5.5$, and galaxies at $0.2 L^{*}<L<0.5 L^{*}$ contributing more than triple the number of UV (and plausibly ionizing) photons as the $L>L^{*}$ galaxies (though both woefully short of the critical value of $\rho_{\mathrm{UV}}$ ). Our observations thus imply that the faint-end slope of the luminosity function at $z \geqslant 6$ is steep, and that the slope is becoming even steeper as $z \rightarrow 8$ (in agreement with previous studies; e.g., Bouwens et al. 2012; Oesch et al. 2012; Bradley et al. 2012).

\subsection{Galaxies Below our Detection Threshold}

Although our observed population appears consistent with multiple lines of evidence pointing to the completion of reionization by, but not too long before, $z=6$, our conclusions are dominated by assumptions about the escape fraction of ionizing photons. Thus, it may be that fainter galaxies do play a large role. Although we will not observe them until the launch of the James Webb Space Telescope (JWST), it is highly likely that there do exist galaxies below our observational limit. However, this introduces another uncertainty, in that we do not know how far down the luminosity function galaxies are able to form.

At some limiting dark matter halo mass, the gas within a halo will no longer be able to self-shield against an ionizing background from the sources which began reionization. In such halos, star formation will be suppressed, effectively cutting off the UV luminosity function at a limiting magnitude $M_{\mathrm{lim}}$ (e.g., Shapiro et al. 1994; Thoul \& Weinberg 1996; Gnedin 2000; Dijkstra et al. 2004b; Okamoto et al. 2008; Finlator et al. 2011), though in the case of inhomogeneous reionization, very small halos may still contribute a significant number of ionizing photons before they are quenched (Finlator et al. 2011). This has not yet been seen observationally, as there is no evidence for a turnover in the luminosity function (e.g., Bouwens et al. 2007, 2011a), which implies that such a cutoff occurs at $M_{\text {lim }}>-16.5$ (the limiting absolute magnitude for $z=4$ galaxies in the HUDF). Such a turnover has also not been seen in large cosmological simulations. For example, the models of Finlator et al. (2011) find an increasing luminosity function down to their resolution limit, which corresponds to $M_{\text {lim }}=-13$. Interestingly, a recent study of high-redshift gamma-ray burst (GRB) host galaxies by Trenti et al. (2012) finds that the non-detection of five $z>5$ GRB hosts implies that galaxies fainter than $M_{\mathrm{UV}}=-15$ were likely present at $z>5$ (see also Tanvir et al. 2012).

The inverted triangles in Figure 3 show the specific UV luminosity density if we take the luminosity functions from the literature and integrate them down to $M_{\text {lim }}=-13$ (Bouwens et al. 2007, 2011a, 2011b; Bradley et al. 2012). The uncertainties on these values were derived via $10^{3}$ Monte Carlo simulations, where in each simulation each Schechter function parameter was modified by a Gaussian random number multiplied by the associated parameter uncertainty.

We note that in these simulations, we have used the published uncertainties on each Schechter function parameter; thus, they were free to vary independent of each other. However, these parameters, particularly the characteristic magnitude $M^{*}$ and the faint-end slope $\alpha$, are correlated, thus the uncertainty on the integrated luminosity functions may be smaller than those derived here. If the published luminosity functions accurately represent the true galaxy population, and the population does indeed extend this faint, than the existing galaxy population at $z=7$ and $z=8$ can sustain reionization for $C / f_{\text {esc }}=10$. However, both luminosity functions are highly uncertain at this point, especially at $z=8$, where the error bar stretches well below the value necessary to sustain reionization. These luminosity function uncertainties, combined with the uncertainty on the value of $M_{\text {lim }}$, render the contribution of the unseen faint galaxies to reionization difficult to assess. 


\subsection{Constraints on the Escape Fraction}

As discussed above, if the escape fraction is modestly high, at $\sim 30 \%$, then the observable galaxy population at $z=6$ can sustain a fully reionized IGM. However, it is highly probable that galaxies significantly fainter than this limit exist, given results from a wide variety of cosmological simulations (e.g., Trenti et al. 2010; Salvaterra et al. 2011; Finlator et al. 2011; Jaacks et al. 2012), and plausible, though scant, observational evidence (e.g., Trenti et al. 2012).

In light of the likelihood that the galaxy luminosity function extends fainter than we can observe, it is instructive to examine the effect of our choice of $30 \%$ for the escape fraction in the above discussion. As shown in Figure 3, our observed galaxy population will yield just enough photons to sustain an ionized IGM at $z=6$ if the escape fraction is $30 \%$. If the luminosity function extends significantly fainter than $M_{\mathrm{UV}}=-18$, and the escape fraction is on average $30 \%$ for all galaxies, then more ionizing photons will be produced than are necessary to complete reionization. In principle, this is not a problem, as once the IGM is reionized, it will stay ionized as long as the critical number of ionizing photons are being produced.

However, there is one observational constraint we have not yet strongly considered, which is the measurement of the ionizing emissivity from observations of the Ly $\alpha$ forest opacity. Bolton \& Haehnelt (2007) studied this at $z=6$ combining simulations with high-resolution spectroscopic observations of $z>6$ quasars and found that only 1.5-3 ionizing photons per hydrogen atom were created, leading them to believe that reionization was "photon-starved." At $z=6$, they calculated a $1 \sigma$ upper limit on the photoionization rate per comoving Mpc, which can be converted to the emission rate of ionizing photons when combined with a measurement of the mean free path of ionizing photons. Using the updated measurement of the mean free path from Songaila \& Cowie (2010), Kuhlen \& FaucherGiguère (2012) find this $1 \sigma$ upper limit on the ionizing photon emission rate to be $<2.6 \times 10^{50} \mathrm{~s}^{-1} \mathrm{Mpc}^{-3}$. This result, though based on a relatively small number of sight lines, implies an upper limit to the number of ionizing photons escaping galaxies at $z=6$.

In Figure 5, we compare this constraint from the Ly $\alpha$ forest to our observations, for a variety of escape fractions. We convert our observed UV luminosity density into a comoving rate of ionizing photons using the same set of stellar population model assumptions used to derive $\epsilon_{53}$ in Section 3.4. Assuming $Z=0.2 Z_{\odot}$, we find $\dot{\mathrm{N}}_{\text {ion }}=1.9 \times 10^{25} \times \rho_{\mathrm{UV}} \times f_{\mathrm{esc}}$. This assumes a star formation history that is in equilibrium (i.e., massive $\mathrm{O}$ stars die as fast as they are formed). We use this to show in Figure 5 the inferred emission rate of ionizing photons from both our observed galaxies (in cyan) and the $z=6$ luminosity function of Bouwens et al. (2008) integrated down to $M_{\mathrm{UV}}=-10$ and -15 (light gray band, where the left and right edges of the band denote $M_{\mathrm{lim}}=-10$ and -15 , respectively).

If $M_{\text {lim }} \sim-18$, an escape fraction of $30 \%$ is allowed within the $2 \sigma$ upper limit on the Ly $\alpha$ forest constraint. With this constraint, the $2 \sigma(1 \sigma)$ upper limit on the escape fraction is $<34 \%$ (17\%). As shown by the light gray band, if the luminosity function extends fainter than our observations, then the escape fraction must decrease, otherwise the $\operatorname{Ly} \alpha$ forest constraints will be violated. Using the limit of $M_{\mathrm{UV}}=-13$ used above in Section 4.4, we find that accounting for galaxies fainter than our detection limit the average escape fraction must be $<13 \%$ $(6 \%)$ at $2 \sigma(1 \sigma)$. This analysis assumes the best-fit luminosity function parameters; if the true $z=6$ luminosity function yields a larger luminosity density it may constrain $f_{\text {esc }}$ even further. We note that the $2 \sigma$ limit on $f_{\text {esc }}$ in both cases (observed and integrated luminosity function) is consistent with sustaining reionization if the clumping factor $C=3$. If future observations constrain the ionizing photon production rate to be closer to the $1 \sigma$ limit, then the observed $z=6$ galaxy population can only sustain reionization if $C \leqslant 2$.

Given the current observations, we can thus say with $95 \%$ confidence that the average escape fraction is $<34 \%$ at $z=6$. Future observations with JWST will push the luminosity functions several magnitudes lower, and thus put more robust constraints on this quantity. We however note that current observations constraining the ionizing background at $z=6$ are due to a relative low number of individual sight lines to distant quasars. Additionally, these quasars reside in biased regions, thus their spectra may not represent a true probe of the mean IGM neutral fraction. Ongoing and future studies such as UKIDDS and VIKING will yield much greater probes of the ionizing background at $z=6-7$, which can then be turned into stronger constraints on the escape fraction of ionizing photons from the $z=6$ galaxy population.

Finally, while the above discussion assumes that the escape fraction represents an average of all galaxies, this does not have to be the case. It is more accurately a luminosity-weighted escape fraction; essentially, this quantity tells us what fraction of the total number of ionizing photons produced in all galaxies escape into the IGM. It may also be the case that the escape fraction is time dependent; ionizing photons may only escape from galaxies due to specific temporal events, such as interactions or starbursts. Our constraints limit the total number of ionizing photons which escape from all galaxies, which can be consistent with a time, luminosity, or mass dependence, or no dependence at all.

\subsection{Constraints on the Reionization History}

As we move into the reionization epoch, and the ionized fraction in the universe becomes less than unity, one can use Equation (2) to calculate the luminosity density necessary to maintain a given ionized fraction by varying the volume ionized fraction $x_{\mathrm{H} \text { II }}$. We can thus indirectly estimate $x_{\mathrm{H} \text { II }}$ as a function of redshift by dividing our observations by the critical value of $\rho_{\mathrm{UV}}$ necessary to sustain a fully ionized IGM as a function of redshift. This approach is a valid approximation to semi-analytic modeling (Madau et al. 1999) because the IGM recombination time remains less than the Hubble time for $C=3$ at $z>6$. This is subject to the same assumptions as the above analysis, including:

1. Galaxies are the only source of ionizing photons in the $z \geqslant 6$ universe. While other sources (i.e., the rare quasar) may exist, their overall contribution is likely negligible.

2. The faint-end cutoff of the luminosity function. As above, we will consider two scenarios: (1) only galaxies above the observational limit of $M_{\mathrm{UV}}=-18$ and (2) the published luminosity functions integrated down to $M_{\mathrm{UV}}=-13$.

3. The clumping factor of ionized hydrogen, which we take to be $C=3$.

4. The escape fraction of ionizing photons; per Section 4.5, we assume here $f_{\text {esc }}=30 \%$ when considering only the observable galaxies and $f_{\text {esc }}=10 \%$ when considering the integrated luminosity functions. Both scenarios are consistent with the Ly $\alpha$ forest constraints and both can sustain a fully reionized IGM at $z=6$ if $C \lesssim 3$.

5. We assume that the ratio $C / f_{\text {esc }}$ does not evolve over the redshifts we study. 

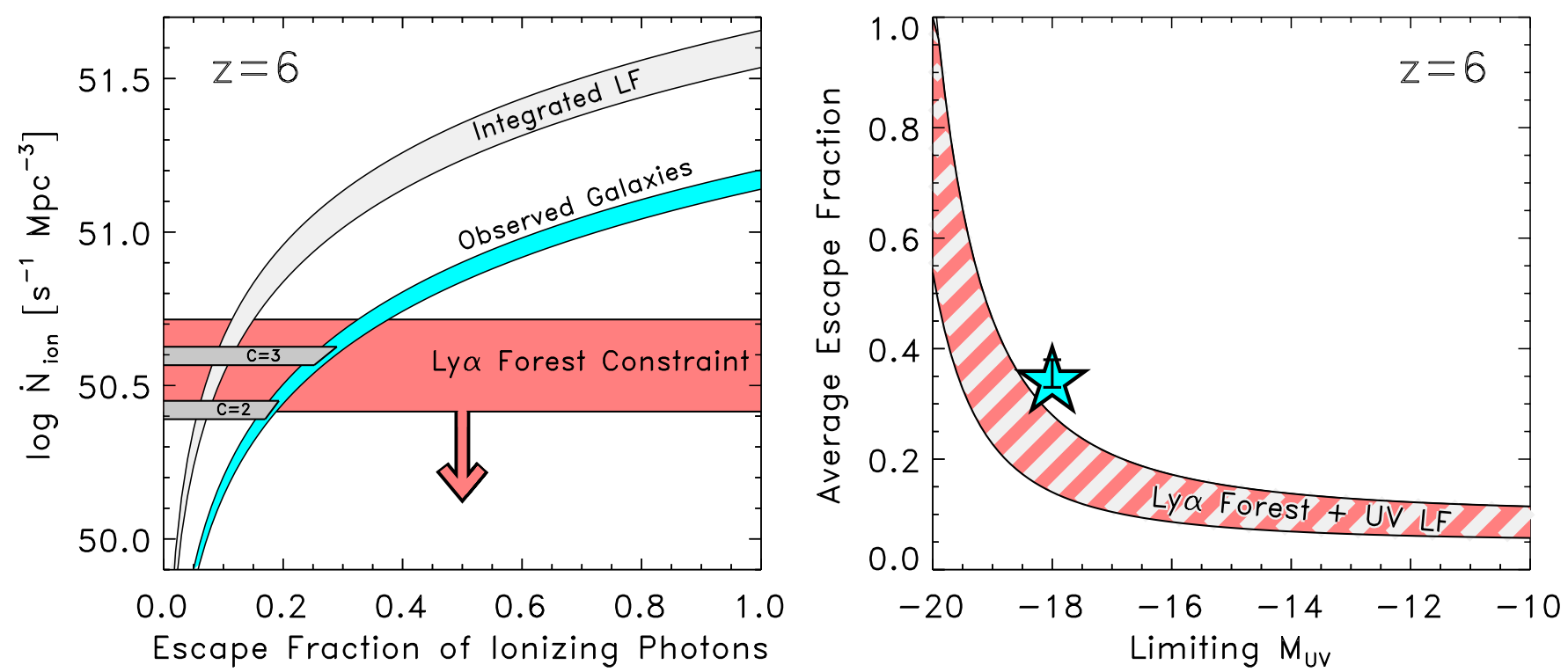

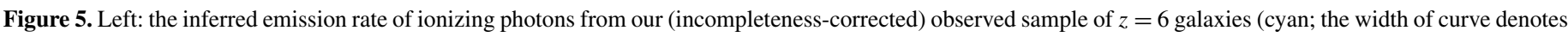

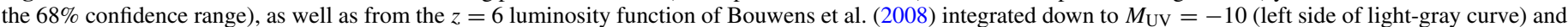

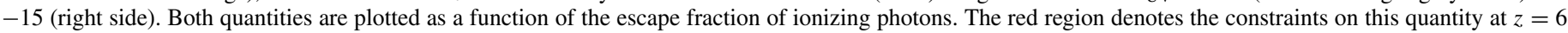

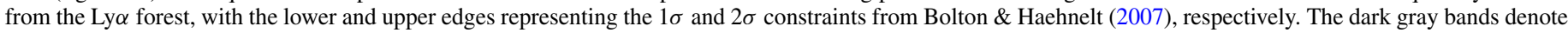

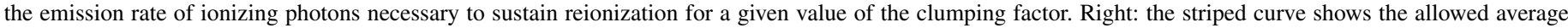

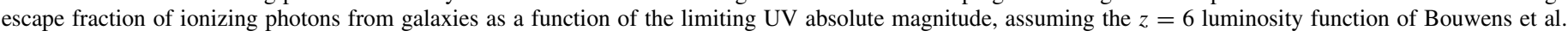

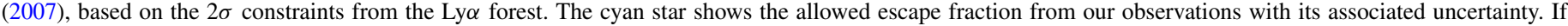

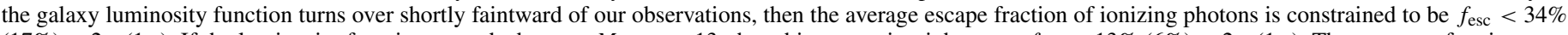

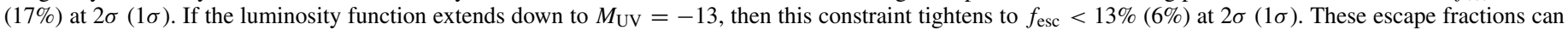
still reionize the IGM if the clumping factor is $\sim 2-3$.

(A color version of this figure is available in the online journal.)

We plot the results of this analysis in Figure 6. Our incompleteness-corrected measurement from the observed galaxies (assuming $f_{\text {esc }}=30 \%$ ) is consistent with a volume ionized fraction of 1.0 at $z=6, \sim 0.4$ at $z=7$, and $\sim 0.2$ at $z=8$. When we consider the integrated luminosity function, with $f_{\text {esc }}=10 \%$, we find a much wider range of possible volume ionized fractions. We note that while the UV luminosity density from the integrated luminosity function will always be larger than that for galaxies above our observational limit, here we assume two different escape fractions for the two magnitude limits, thus their ionizing luminosity densities are comparable. At $z=6$, the integrated luminosity functions are consistent with ionized fractions of $\sim 0.7-1.0$. At $z=7$ and 8 , this is reduced to $0.3-1.0$ and $0.1-0.95$, respectively. The very wide range of possible ionized fractions from the integrated luminosity functions are due to the increasingly larger uncertainties in the Schechter function parameters as one pushes to higher redshift.

Also in Figure 6, we plot a number of results from the literature, including constraints on the volume ionized fraction from quasars at $z \leqslant 6$ (Fan et al. 2006) and $z=7$ (Bolton et al. 2011), WMAP (Komatsu et al. 2011), Ly $\alpha$ emitter luminosity functions (Ouchi et al. 2010), and Ly $\alpha$ spectroscopic studies (Pentericci et al. 2011; Schenker et al. 2012; Ono et al. 2012). We note that results from the latter are very preliminary, as thus far only small numbers of $z \gtrsim 7$ galaxies have spectroscopically observed Ly $\alpha$ emission. Both our observed galaxy population and the integrated luminosity functions are consistent with this wide and complementary range of reionization probes. The only exception is the single known quasar at $z=7$, which has a near-zone size consistent with an ionized fraction of $\lesssim 90 \%$ and appears slightly inconsistent with our observed galaxy population (though consistent with the integrated luminosity functions). However, this is but a single sight line to the only currently known $z \geqslant 7$ quasar, and thus many more sight lines are needed to truly constrain the IGM from this type of probe.

Our new observations combined with the previous, complementary probes of the ionization state of the IGM show a consistent reionization picture, where the ionized fraction in the IGM is essentially unity at $z \leqslant 6$. At $z \sim 7$, there is a hint from a number of lines of evidence (including our observed galaxy population) that the ionized fraction may dip below unity. The integrated luminosity function prefers an ionized fraction less than unity, but it is consistent with a fully ionized IGM at $1 \sigma$. This is not the case at $z=8$, where galaxies are the only probe we currently have, and they seem to indicate an ionized fraction of $<80 \%-90 \%$ (unless the escape fraction in all galaxies is unity, which would indicate extreme evolution from $z=6$, and is unlikely; see Section 4.5). Unfortunately, more robust constraints on the evolution of $x_{\mathrm{H} \text { II }}$ from the integrated luminosity functions are difficult due to the uncertainty in the Schechter function parameters. We note that we have used the uncertainties on each Schechter function parameter to compute the uncertainty in the integrated luminosity function (see Section 4.4 for more details). These parameters, particularly the characteristic magnitude $M^{*}$ and the faint-end slope $\alpha$, are known to be correlated, thus the uncertainty on the integrated luminosity functions may be smaller than those derived here. In any case, at $z \geqslant 7$, the faint-end slope is not well constrained, which is responsible for the bulk of the uncertainty on $x_{\mathrm{HII}}$. More robust luminosity functions in this epoch will help decrease the uncertainty on $x_{\mathrm{H} \text { II }}$ from galaxy measurements.

Finally, in Figure 6 we also show the concordance models of Kuhlen \& Faucher-Giguère (2012), which present a picture of the reionization history of the universe consistent with the 


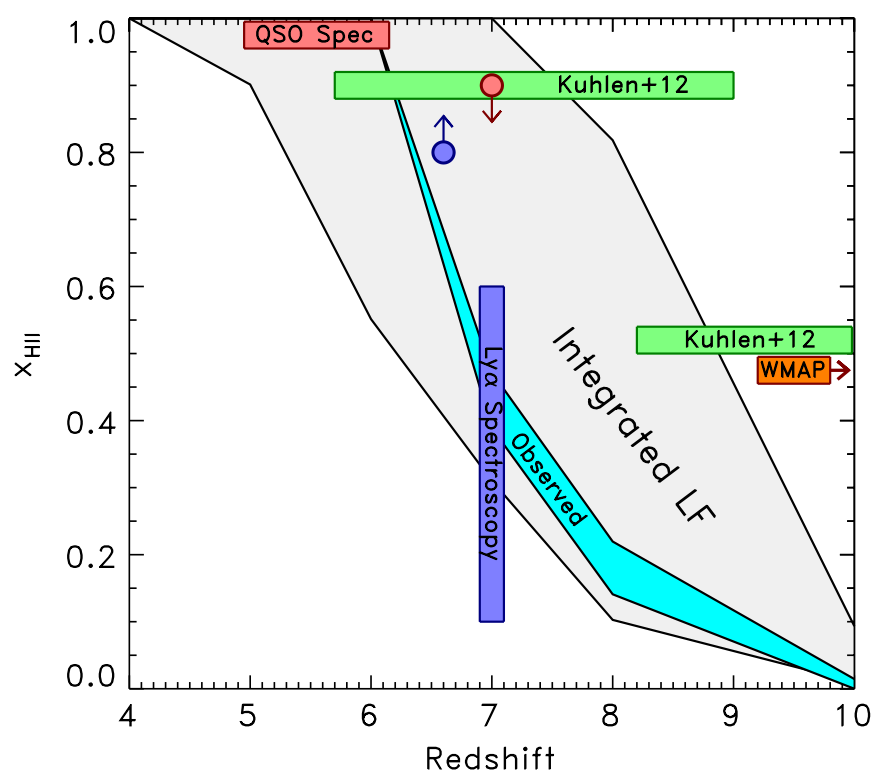

Figure 6. Volume ionized fraction, $x_{\mathrm{H}}$, of the IGM which can be sustained given the observed UV luminosity densities (and our assumptions on $\mathrm{C}$ and $f_{\mathrm{esc}}$ ) as a function of redshift. We assume that galaxies are the only significant sources of ionizing photons in this epoch. The cyan and gray curves denote the value of $x_{\mathrm{H}}$ inferred from comparing our observed galaxies, and the integrated luminosity functions, respectively, to the critical luminosity density from Equation (2). We have assumed $C / f_{\text {esc }}=10$ when only considering the observable galaxies, and $C / f_{\text {esc }}=30$ when considering the integrated luminosity functions (both of which are consistent with limits on $f_{\text {esc }}$ from the $z=6$ Ly $\alpha$ forest presented in Section 4.5 , for $C=3$ ). We note that while the luminosity density from the integrated luminosity functions will always be larger than that for galaxies above our observational limit, here the two different escape fractions for the two magnitude limits result in their ionizing luminosity densities being comparable. We plot constraints on $x_{\mathrm{H}}$ from spectroscopy of quasars at $z<6$ from Fan et al. (2006) and at $z=7$ from Bolton et al. (2011) in red. The blue circle denotes constraints on $x_{\mathrm{H} \text { II }}$ from the evolution in the Ly $\alpha$ luminosity function from $z=$ 5.7 to 6.6 from Ouchi et al. (2010), while the blue bar denotes the range of $x_{\mathrm{HII}}$ values inferred from the $z=7$ follow-up Ly $\alpha$ spectroscopic studies of Pentericci et al. (2011), Schenker et al. (2012), and Ono et al. (2012). The instantaneous redshift for reionization from WMAP $(10.6 \pm 1.2)$ is indicated by the orange rectangle. The derived $50 \%$ and $90 \% x_{\mathrm{H}_{\text {II }}}$ redshifts from the study of Kuhlen \& Faucher-Giguère (2012) are shown in green, where the width of the green bars denote the $1 \sigma$ uncertainty (from Figure 9 of Kuhlen \& Faucher-Giguère). Our observations, which are consistent with a wide range of other, complementary, approaches, find a picture where the universe is fully ionized by $z=6$, with the neutral fraction becoming non-negligible at $z \gtrsim 7$ for our assumptions regarding $C / f_{\text {esc }}$.

(A color version of this figure is available in the online journal.)

Ly $\alpha$ forest, WMAP optical depth, and the kSZ effect. They also fold in the observed galaxy luminosity functions, though in this case they assume that the luminosity function parameters evolve smoothly with redshift; we do not know if this is truly the case, particularly at $z>8$, beyond which the strong dropoff in the observed luminosity density is intriguing. We plot their results for the redshift of a $50 \%$ and $90 \%$ ionized fraction. Comparing our inferred values of $x_{\mathrm{H} \text { II }}$ from galaxies with their conclusions, we find general agreement at $z<8$, in that they find that reionization completes by $z=6$ and that it is $90 \%$ complete at $z=7.5 \pm 1.5$.

However, at higher redshift, there appears to be some tension between our observations and their results, in that the Kuhlen \& Faucher-Giguère (2012) study prefers a 50\% reionized universe at a higher redshift than appears possible with the observations, though it is marginally consistent with the integrated luminosity functions. However, we stress that the results from Kuhlen \& Faucher-Giguère (2012) were tuned to match both the observed luminosity functions and the WMAP constraint on the optical depth to Thomson scattering whereas our results use only the luminosity functions. Given that the WMAP constraint prefers a redshift of instantaneous reionization of $10.6 \pm 1.2$, it is not surprising that observations showing a strong decline in the galaxy UV luminosity density at $z \geqslant 8$ would underproduce the electron optical depth. This can be reconciled by allowing the escape fraction and/or the limiting magnitude to evolve with redshift, as is done by Kuhlen \& Faucher-Giguère (2012). This would imply that very faint galaxies may be necessary to drive reionization at the earliest stages, and that reionization gets its start at much higher redshifts (i.e., $z>10$ ). We point out that the $z=8$ luminosity function and the limits on the $z=10$ luminosity function are very rough at this point in time. Future observations, likely with JWST (see Section 4.7), will greatly improve constraints on the luminosity functions in this early epoch, and at that time we can learn more about whether the contribution of galaxies to the early stages of reionization is consistent with all other constraints.

\subsection{Implications for Future Surveys}

At $z=6$, our observations (shown in Figures 3 and 4) already reveal a population of galaxies capable of sustaining reionization, while at $z=7$ and 8 the observed galaxy population falls short. Our current observations are however susceptible to the uncertainty about what lies below our detection threshold, as well as our assumptions about the escape fraction. The former can potentially be alleviated by integrating down the luminosity function, as we have done in Section 4.4, but this yields its own uncertainty: How faint does the UV luminosity function continue?

In order to measure this, we will require the deeper observations only possible with $J W S T$. In a hypothetical $\sim 800 \mathrm{hr}$ program, JWST can reach $m_{\mathrm{AB}}(5 \sigma)=31.5$ mag over a single pointing in four bands: F070W, F090W, F115W, and F150W, which can be used to select samples at similar redshifts as in our study. ${ }^{14}$ Is this deep enough to probe faint enough values of $M_{\text {lim }}$ to conclusively find the reionizing population at $z=7$ and 8 ? To investigate this, we created Figure 7, where we plot the limiting absolute UV magnitude necessary to sustain reionization for a given ratio of $C / f_{\text {esc }}$, assuming the luminosity functions from the literature (see Wilkins et al. 2011a for a similar analysis at $z=7)$.

In this figure, we show the limiting magnitude of our HST study, as well as that of the hypothetical JWST deep field, where each colored line denotes the value of $M_{\text {lim }}$ and $C / f$ esc necessary to sustain reionization for a given redshift. As we discussed above, for our assumption of $C / f_{\text {esc }}=10$, our $z=$ 6 observed galaxy population is sufficient to sustain a reionized IGM. In fact, this figure shows that the observed population can reionize the IGM for values of $C / f_{\text {esc }} \lesssim 14$. At $z=6, J W S T$ will trace the rest-frame UV luminosity function down to faint levels, enabling the search for a turnover at faint magnitudes down to $M_{1500}=-15.3$. If the luminosity function continues its steep climb to this magnitude, then as shown in Figure 5, the average escape fraction for galaxies will be constrained to be less than $\sim 15 \%$.

At $z=7$, galaxies brighter than HSTs magnitude limit can only reionize the universe if $C / f_{\text {esc }} \lesssim 6$, which, for $C \geqslant 3$, necessitates an escape fraction $\geqslant 50 \%$. While this is possible, there is no strong observational evidence yet to support such

\footnotetext{
14 http://jwstetc.stsci.edu/etc/input/nircam/imaging/
} 


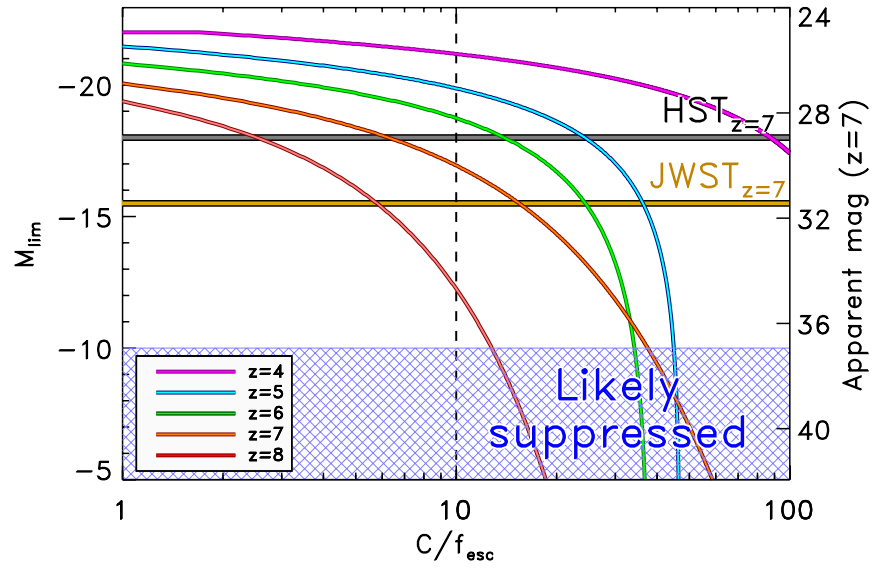

Figure 7. Colored curves show the limiting UV absolute magnitude for different redshifts necessary to sustain reionization for a given ratio between the IGM clumping factor and the ionizing escape fraction. The hatched region denotes the magnitude range where star formation is likely to be suppressed via photoheating from the sources which began the process of reionization at higher redshift. The dashed line shows our canonical model of a clumping factor of three and $f_{\text {esc }}=30 \%$, or $C / f_{\text {esc }}=10$, which is a reasonable assumption for the conditions in the universe at $z \geqslant 6$. The horizontal gray bar denotes the limiting UV magnitude of $H S T$ observations of the HUDF at $z=7\left(m_{\mathrm{AB}}=\right.$ 29). A hypothetical JWST deep field will reach 2.5 mag deeper to $m_{\mathrm{AB}}=31.5$ or $M_{\mathrm{UV}}=-15.5$ (for $z=7$ ). At $z=6$, our observations (shown in Figure 3) already reveal a population of galaxies capable of sustaining reionization. At $z=7$, the observed population is not bright enough to sustain reionization, thus if reionization completes at $z>6$, fainter galaxies are necessary, which should be revealed by JWST. At $z=8$, even JWST cannot image deep enough to see the hypothetical extremely faint population necessary to complete reionization at $z=8$.

(A color version of this figure is available in the online journal.)

a scenario. However, JWST will reach deep enough, down to $M_{\text {lim }}=-15.5$; if the luminosity function continues its steep slope down to such faint magnitudes, and $C / f_{\text {esc }} \lesssim 15$, then galaxies could sustain a reionized IGM by $z=7$ (see also Salvaterra et al. 2011). At $z=8$, even JWST cannot probe deep enough to see the limiting magnitude necessary to reionize the IGM (again assuming $C / f_{\text {esc }} \geqslant 10$ ). If the universe were reionized by $z=8$, it necessitates a luminosity function that continues with a steep slope $(\alpha \sim-2)$ down to $M_{\text {lim }}=-12$. However, such a result would create tension with the observed dearth of $\operatorname{Ly} \alpha$ emission and the small size of quasar near zones at $z=7$ (see Section 4.2). This tension could be resolved if the escape fraction of galaxies is luminosity dependent, and galaxies with $L \ll L^{*}$ have very low escape fractions, which could be the case if they are highly gas dominated (e.g., Gnedin et al. 2008).

\section{CONCLUSIONS}

We have measured the specific UV luminosity density ( $\left.\rho_{\mathrm{UV}}\right)$ from a large sample of galaxies at $z=6,7$, and 8 . Using a theoretical model to convert from UV to ionizing photons, and determining the level of ionizing photons necessary to sustain a fully reionized IGM, we have investigated the contribution of observed galaxies to the reionization of the IGM. A large body of work over the past $\sim$ decade points to a probable low clumping factor. Thus, previous surveys which assumed a large clumping factor and deduced that galaxies could not reionize the universe may have reached the opposite conclusion today. Unfortunately, not much is known about the escape fraction of ionizing photons at high redshift, but current observations are consistent with a rising escape fraction with increasing redshift or at least an increased incidence of large escape fractions. With our new sample, and our increased understanding of the process of reionization, we have reached the following conclusions.

1. We measure $\rho_{\mathrm{UV}}$ at $z=6$ to be $0.79 \pm 0.06 \times$ $10^{26} \mathrm{erg} \mathrm{s}^{-1} \mathrm{~Hz}^{-1} \mathrm{Mpc}^{-3}$ for the observed population of galaxies; this is much more robust than previous measures, which had much smaller samples and were limited to single band detections, and differed by a factor of $>5$. Additionally, this result is independent of a Schechter function parameterization for the form of the luminosity function. Assuming $C / f_{\text {esc }} \sim 10$, we find that the observed galaxy population at $z=6$ is sufficient to sustain a fully reionized IGM. In contrast to many previous studies, this result does not need to invoke galaxies fainter than our detection threshold.

2. We measure $\rho_{\mathrm{UV}}$ of the observed galaxy population at $z=7$ and 8 to be $0.49 \pm 0.11$ and $0.27 \pm 0.06 \times$ $10^{26} \mathrm{erg} \mathrm{s}^{-1} \mathrm{~Hz}^{-1} \mathrm{Mpc}^{-3}$, respectively. These values fall short of what is needed to reionize the IGM. While fainter galaxies may render the population capable of completing reionization, even JWST will not go deep enough to see the necessary limiting magnitude at $z=8$.

3 . If we only consider the observed galaxies, we find a scenario where though $z=7$ and 8 galaxies contribute to reionization, the IGM only becomes fully reionized by $z=6$. This is consistent with independent studies of both Ly $\alpha$ emission from normal galaxies, and the study of Ly $\alpha$ absorption in the near-zone of the $z=7$ quasar, both of which find that the IGM at $z=7$ likely has a neutral fraction $\geqslant 10 \%$.

4. Accounting for constraints on the total emission rate of ionizing photons from measurements of the Ly $\alpha$ forest at $z=6$, we find that if our observed galaxies represent the total galaxy population, then the average $z=6$ escape fraction must be $f_{\text {esc }}<34 \%$. However, fainter galaxies likely do exist, and if the luminosity function extends down to $M_{\text {lim }}=-13$, then the average escape fraction constraint tightens to $f_{\text {esc }}<13 \%$. These escape fractions can sustain reionization at $z=6$ either with our observed galaxies or with the integrated luminosity function, for values of the clumping factor $\leqslant 3$.

5. We investigate the evolution of the volume ionized fraction $x_{\mathrm{H} \text { II }}$ of the IGM. We do this by combining the observed galaxy population of our study and the luminosity functions from the literature with limits on the escape fraction considering both limiting magnitudes. While the IGM appears to be fully ionized by $z \leqslant 6$, the volume ionized fraction may drop below unity by $z=7$, consistent with a number of complementary analyses. Considering only the (roughly known) contribution from galaxies at $z \geqslant 8$, the volume ionized fraction may drop substantially below unity, though this is in mild tension with reionization models which incorporate the optical depth due to electron scattering, which was not accounted for in our analysis. Better constraints on the $z \geqslant 8$ luminosity functions, likely from $J W S T$, will place stronger constraints on the reionization history at that early time. If the strong drop in the luminosity density at $z>8$ is verified, it may imply that some additional source of ionizing photons at $z>10$ is warranted to remain consistent with the WMAP-measured Thomson scattering optical depth to electrons.

We conclude that in order to make progress on the issue of when and how reionization happened, we need better constraints 
on the escape fraction of ionizing photons or the limiting magnitude of the UV luminosity function. As these quantities are correlated due to the constraints from the Ly $\alpha$ forest, constraints on one will yield more robust constraints on the other. While direct measurement of the escape fraction at high redshift is not possible, much deeper observations with JWST should confirm whether the luminosity function extends down to at least $M_{\text {lim }}=-15.5$, which will place stronger constraints on the escape fraction at $z=6$, and uncover whether the $z=7$ galaxy population is sufficient to sustain an ionized universe.

We thank Volker Bromm, Andrea Ferrara, and Milos Milosavljevic for stimulating conversations, as well as Chris Conselice, Yan Gong, Bahram Mobasher, Brian Siana, and Matt McQuinn for useful comments. Support for S.L.F. was provided by NASA through Hubble Fellowship grant HST-HF51288.01. C.P. was supported in part by HST program 12060. These grants were all awarded by the Space Telescope Science Institute, which is operated by the Association of Universities for Research in Astronomy, Inc., for NASA, under contract NAS 5-26555. J.S.D. acknowledges the support of the European Research Council through an Advanced Grant and the support of the Royal Society via a Wolfson Research Merit award.

\section{REFERENCES}

Anderson, J., \& Bedin, L. R. 2010, PASP, 122, 1035

Becker, G. D., Rauch, M., \& Sargent, W. L. W. 2007, ApJ, 662, 72

Bertin, E., \& Arnouts, S. 1996, A\&AS, 117, 393

Bolton, J. S., \& Haehnelt, M. G. 2007, MNRAS, 382, 325

Bolton, J. S., Haehnelt, M. G., Warren, S. J., et al. 2011, MNRAS, 416, L70

Bouwens, R. J., Illingworth, G. D., Blakeslee, J. P., \& Franx, M. 2006, ApJ, 653,53

Bouwens, R. J., Illingworth, G. D., Franx, M., \& Ford, H. 2007, ApJ, 670, 928 Bouwens, R. J., Illingworth, G. D., Franx, M., \& Ford, H. 2008, ApJ, 686, 230 Bouwens, R. J., Illingworth, G. D., Oesch, P. A., et al. 2010, ApJ, 708, L69 Bouwens, R. J., Illingworth, G. D., Oesch, P. A., et al. 2012, ApJ, 752, L5 Bouwens, R. J., Illingworth, G. D., Oesch, P. A., et al. 2011a, ApJ, 737, 90 Bouwens, R. J., Illingworth, G. D., Labbe, I., et al. 2011b, Nature, 469, 504 Bradley, L. D., Trenti, M., Oesch, P. A., et al. 2012, arXiv:1204.3641 Brammer, G. B., van Dokkum, P. G., \& Coppi, P. 2008, ApJ, 686, 1503 Bromm, V., Kudritzki, R. P., \& Loeb, A. 2001, ApJ, 552, 464 Bromm, V., \& Larson, R. B. 2004, ARA\&A, 42, 79

Bruzual, G., \& Charlot, S. 2003, MNRAS, 344, 1000

Bunker, A. J., Stanway, E. R., Ellis, R. S., \& McMahon, R. G. 2004, MNRAS, 355,374

Bunker, A. J., Wilkins, S., Ellis, R. S., et al. 2010, MNRAS, 409, 855

Calzetti, D., Kinney, A. L., \& Storchi-Bergmann, T. 1994, ApJ, 429, 582 Chary, R.-R. 2008, ApJ, 680, 32

Choudhury, T. R., Ferrara, A., \& Gallerani, S. 2008, MNRAS, 385, L58

Clark, P. C., Glover, S. C. O., Klessen, R. S., \& Bromm, V. 2011, ApJ, 727, 110

Conroy, C., \& Kratter, K. 2012, ApJ, 755, 123

Davé, R., Cen, R., Ostriker, J. P., et al. 2001, ApJ, 552, 473

Dijkstra, M., Haiman, Z., \& Loeb, A. 2004a, ApJ, 613, 646

Dijkstra, M., Haiman, Z., Rees, M. J., \& Weinberg, D. H. 2004b, ApJ, 601, 666 Fan, X., Strauss, M. A., Becker, R. H., et al. 2006, AJ, 132, 117

Faucher-Giguère, C.-A., Lidz, A., Hernquist, L., \& Zaldarriaga, M. 2008, ApJ, 688,85

Ferguson, H. C., Dickinson, M., Giavalisco, M., et al. 2004, ApJ, 600, L107 Fernandez, E. R., \& Shull, J. M. 2011, ApJ, 731, 20

Finkelstein, K. D., Papovich, C., Finkelstein, S. L., et al. 2011, ApJ, 742, 108

Finkelstein, S. L., Papovich, C., Giavalisco, M., et al. 2010, ApJ, 719, 1250

Finkelstein, S. L., Papovich, C., Salmon, B., et al. 2012, ApJ, 756, 164

Finlator, K., Davé, R., \& Özel, F. 2011, ApJ, 743, 169

Finlator, K., Oh, S. P., Özel, F., \& Davé, R. 2012, MNRAS, in press (arXiv:1209.2489)

Fontana, A., Vanzella, E., Pentericci, L., et al. 2010, ApJ, 725, L205

Giavalisco, M., Dickinson, M., Ferguson, H. C., et al. 2004, ApJ, 600, L103

Glover, S. 2005, Space Sci. Rev., 117, 445

Gnedin, N. Y. 2000, ApJ, 542, 535

Gnedin, N. Y., Kravtsov, A. V., \& Chen, H.-W. 2008, ApJ, 672, 765
Gnedin, N. Y., \& Ostriker, J. P. 1997, ApJ, 486, 581

González, V., Labbé, I., Bouwens, R. J., et al. 2010, ApJ, 713, 115

Grazian, A., Castellano, M., Koekemoer, A. M., et al. 2011, A\&A, 532, A33

Greif, T. H., \& Bromm, V. 2006, MNRAS, 373, 128

Greif, T. H., Bromm, V., Clark, P. C., et al. 2012, ApJ, 751, 51

Greif, T. H., Springel, V., White, S. D. M., et al. 2011, ApJ, 737, 75

Grogin, N. A., Kocevski, D. D., Faber, S. M., et al. 2011, ApJS, 197, 35

Haardt, F., \& Madau, P. 2012, ApJ, 746, 125

Hopkins, P. F., Richards, G. T., \& Hernquist, L. 2007, ApJ, 654, 731

Hu, E. M., Cowie, L. L., Barger, A. J., et al. 2010, ApJ, 725, 394

Iliev, I. T., Mellema, G., Pen, U.-L., et al. 2006, MNRAS, 369, 1625

Inoue, A. K., Iwata, I., \& Deharveng, J.-M. 2006, MNRAS, 371, L1

Iwata, I., Inoue, A. K., Matsuda, Y., et al. 2009, ApJ, 692, 1287

Jaacks, J., Choi, J.-H., Nagamine, K., Thompson, R., \& Varghese, S. 2012, MNRAS, 420, 1606

Jones, T., Stark, D. P., \& Ellis, R. S. 2011, arXiv e-prints

Koekemoer, A. M., Faber, S. M., Ferguson, H. C., et al. 2011, ApJS, 197, 36

Komatsu, E., Smith, K. M., Dunkley, J., et al. 2011, ApJS, 192, 18

Kornei, K. A., Shapley, A. E., Martin, C. L., et al. 2012, arXiv:1205.0812

Kuhlen, M., \& Faucher-Giguère, C.-A. 2012, MNRAS, 423, 862

Kulkarni, G., \& Choudhury, T. R. 2011, MNRAS, 412, 2781

Labbé, I., González, V., Bouwens, R. J., et al. 2010, ApJ, 716, L103

Lorenzoni, S., Bunker, A. J., Wilkins, S. M., et al. 2011, MNRAS, 414, 1455

Madau, P., Haardt, F., \& Rees, M. J. 1999, ApJ, 514, 648

Malhotra, S., \& Rhoads, J. E. 2004, ApJ, 617, L5

McGreer, I. D., Mesinger, A., \& Fan, X. 2011, MNRAS, 415, 3237

McLure, R. J., Cirasuolo, M., Dunlop, J. S., Foucaud, S., \& Almaini, O. 2009, MNRAS, 395, 2196

McLure, R. J., Dunlop, J. S., Cirasuolo, M., et al. 2010, MNRAS, 403, 960 McLure, R. J., Dunlop, J. S., de Ravel, L., et al. 2011, MNRAS, 418, 2074 McQuinn, M. 2012, arXiv:1206.1335

McQuinn, M., Oh, S. P., \& Faucher-Giguère, C.-A. 2011, ApJ, 743, 82

Mesinger, A. 2010, MNRAS, 407, 1328

Mesinger, A., McQuinn, M., \& Spergel, D. N. 2012, MNRAS, 422, 1403

Meurer, G. R., Heckman, T. M., \& Calzetti, D. 1999, ApJ, 521, 64

Miralda-Escudé, J., Haehnelt, M., \& Rees, M. J. 2000, ApJ, 530, 1

Mortlock, D. J., Warren, S. J., Venemans, B. P., et al. 2011, Nature, 474, 616

Muñoz, J. A., \& Loeb, A. 2011, ApJ, 729, 99

Nestor, D. B., Shapley, A. E., Steidel, C. C., \& Siana, B. 2011, ApJ, 736, 18

Oesch, P. A., Bouwens, R. J., Carollo, C. M., et al. 2010a, ApJ, 709, L21

Oesch, P. A., Bouwens, R. J., Illingworth, G. D., et al. 2010b, ApJ, 709, L16

Oesch, P. A., Bouwens, R. J., Illingworth, G. D., et al. 2012, arXiv:1201.0755

Okamoto, T., Gao, L., \& Theuns, T. 2008, MNRAS, 390, 920

Oke, J. B., \& Gunn, J. E. 1983, ApJ, 266, 713

Ono, Y., Ouchi, M., Mobasher, B., et al. 2012, ApJ, 744, 83

Ouchi, M., Shimasaku, K., Furusawa, H., et al. 2010, ApJ, 723, 869

Pawlik, A. H., Schaye, J., \& van Scherpenzeel, E. 2009, MNRAS, 394, 1812

Peng, C. Y., Ho, L. C., Impey, C. D., \& Rix, H.-W. 2002, AJ, 124, 266

Pentericci, L., Fontana, A., Vanzella, E., et al. 2011, ApJ, 743, 132

Pritchard, J. R., Loeb, A., \& Wyithe, J. S. B. 2010, MNRAS, 408, 57

Raičević, M., \& Theuns, T. 2011, MNRAS, 412, L16

Razoumov, A. O., \& Sommer-Larsen, J. 2010, ApJ, 710, 1239

Ricotti, M., \& Shull, J. M. 2000, ApJ, 542, 548

Robertson, B. E., Ellis, R. S., Dunlop, J. S., McLure, R. J., \& Stark, D. P. 2010, Nature, 468, 49

Ryan, R., Yan, H., \& Finkelstein, S. L. 2012, ApJ, submitted

Salvaterra, R., Ferrara, A., \& Dayal, P. 2011, MNRAS, 414, 847

Schaerer, D. 2002, A\&A, 382, 28

Schenker, M. A., Stark, D. P., Ellis, R. S., et al. 2012, ApJ, 744, 179

Shapiro, P. R., Giroux, M. L., \& Babul, A. 1994, ApJ, 427, 25

Shapley, A. E., Steidel, C. C., Pettini, M., \& Adelberger, K. L. 2003, ApJ, 588, 65

Shapley, A. E., Steidel, C. C., Pettini, M., Adelberger, K. L., \& Erb, D. K. 2006, ApJ, 651, 688

Shull, J. M., Harness, A., Trenti, M., \& Smith, B. D. 2012, ApJ, 747, 100

Siana, B., Teplitz, H. I., Colbert, J., et al. 2007, ApJ, 668, 62

Siana, B., Teplitz, H. I., Ferguson, H. C., et al. 2010, ApJ, 723, 241

Songaila, A., \& Cowie, L. L. 2010, ApJ, 721, 1448

Stark, D. P., Ellis, R. S., Bunker, A., et al. 2009, ApJ, 697, 1493

Stark, D. P., Ellis, R. S., Chiu, K., Ouchi, M., \& Bunker, A. 2010, MNRAS, 408, 1628

Stark, D. P., Ellis, R. S., \& Ouchi, M. 2011, ApJ, 728, L2

Steidel, C. C., Adelberger, K. L., Giavalisco, M., Dickinson, M., \& Pettini, M. 1999, ApJ, 519, 1

Steidel, C. C., Pettini, M., \& Adelberger, K. L. 2001, ApJ, 546, 665 
Tanvir, N. R., Levan, A. J., Fruchter, A. S., et al. 2012, ApJ, 754, 46

Thoul, A. A., \& Weinberg, D. H. 1996, ApJ, 465, 608

Trenti, M., Bradley, L. D., Stiavelli, M., et al. 2011, ApJ, 727, L39

Trenti, M., Perna, R., Levesque, E. M., Shull, J. M., \& Stocke, J. T. 2012, ApJ, 749, L38

Trenti, M., Stiavelli, M., Bouwens, R. J., et al. 2010, ApJ, 714, L202

Treu, T., Trenti, M., Stiavelli, M., Auger, M. W., \& Bradley, L. D. 2012, ApJ, 747,27

Tumlinson, J., \& Shull, J. M. 2000, ApJ, 528, L65

Vanzella, E., Giavalisco, M., Inoue, A. K., et al. 2010, ApJ, 725, 1011

Vanzella, E., Guo, Y., Giavalisco, M., et al. 2012, MNRAS, 424, 54

Wilkins, S. M., Bunker, A. J., Lorenzoni, S., \& Caruana, J. 2011a, MNRAS, 411,23
Wilkins, S. M., Bunker, A. J., Stanway, E., Lorenzoni, S., \& Caruana, J 2011b, MNRAS, 417, 717

Willott, C. J., Delorme, P., Reylé, C., et al. 2010, AJ, 139, 906

Windhorst, R. A., Cohen, S. H., Hathi, N. P., et al. 2011, ApJS, 193, 27

Wise, J. H., \& Abel, T. 2005, ApJ, 629, 615

Wise, J. H., \& Cen, R. 2009, ApJ, 693, 984

Wise, J. H., Turk, M. J., Norman, M. L., \& Abel, T. 2012, ApJ, 745, 50

Yajima, H., Choi, J.-H., \& Nagamine, K. 2011, MNRAS, 412, 411

Yajima, H., Umemura, M., Mori, M., \& Nakamoto, T. 2009, MNRAS, 398, 715

Yan, H., \& Windhorst, R. A. 2004, ApJ, 600, L1

Yan, H.-J., Windhorst, R. A., Hathi, N. P., et al. 2010, Res. Astron. Astrophys., 10,867

Zahn, O., Reichardt, C. L., Shaw, L., et al. 2012, ApJ, 756, 65 